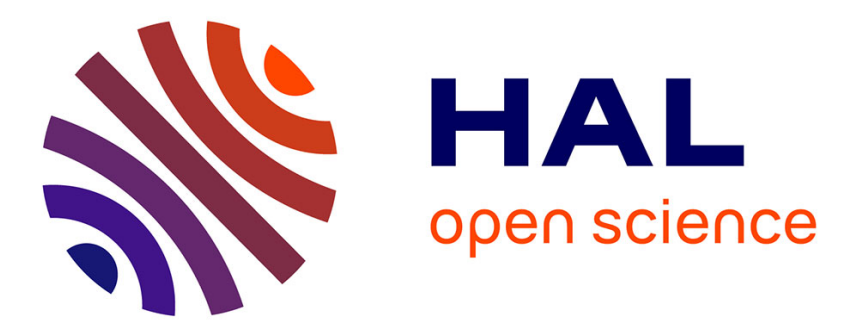

\title{
Cooperative GNSS Positioning aided by Road-Features Measurements
}

Luís Conde Bento, Philippe Bonnifait, Urbano Nunes

\section{To cite this version:}

Luís Conde Bento, Philippe Bonnifait, Urbano Nunes. Cooperative GNSS Positioning aided by RoadFeatures Measurements. Transportation research. Part C, Emerging technologies, 2017, 79, pp.42-57. 10.1016/j.trc.2017.01.002 . hal-01494221

\section{HAL Id: hal-01494221 \\ https://hal.science/hal-01494221}

Submitted on 22 Mar 2017

HAL is a multi-disciplinary open access archive for the deposit and dissemination of scientific research documents, whether they are published or not. The documents may come from teaching and research institutions in France or abroad, or from public or private research centers.
L'archive ouverte pluridisciplinaire HAL, est destinée au dépôt et à la diffusion de documents scientifiques de niveau recherche, publiés ou non, émanant des établissements d'enseignement et de recherche français ou étrangers, des laboratoires publics ou privés. 


\title{
Cooperative GNSS Positioning aided by Road-Features Measurements
}

\author{
Luís Conde Bento ${ }^{\mathrm{a}, \mathrm{c}}$, Philippe Bonnifait ${ }^{\mathrm{b}}$, Urbano Nunes ${ }^{\mathrm{a}}$ \\ ${ }^{a}$ ISR-Institute of Systems and Robotics, Electrical and Computer Eng. Department, University of Coimbra, Portugal. \\ ${ }^{b}$ Heudiasyc UMR CNRS 7253, Sorbonne Universités, Université de Technologie de Compiègne, France. \\ ${ }^{c}$ ESTG, Polytechnic Institute of Leiria, Portugal.
}

\begin{abstract}
Cooperation between road users trough V2X communication is a way to improve GNSS localization accuracy. When vehicles localization systems involve standalone GNSS receivers, the resulting accuracy can be affected by satellite-specific errors of several meters. This paper studies how road-features like lane marking detected by on-board cameras can be exploited to reduce absolute position errors of cooperative vehicles sharing information in real-time in a network. The algorithms considered in this work are based on a error bounded set membership strategy. In every vehicle, a set membership algorithm computes the absolute position and an estimation of the satellite-specific errors by using raw GNSS pseudoranges, lane boundary measurements and a 2D georeferenced road map which provides absolute geometric constraints. As lane-boundary measurements provide essentially cross-track corrections in the position estimation process, cooperation enables the vehicles to improve their own estimates thanks to the different orientation of the roads. Set-membership methods are very efficient to solve this problem since they don't involve any independence hypothesis of the errors and so, the same information can be used several times in the computation. Such class of algorithm provides a novel approach to improve position accuracy for connected vehicles guaranteeing the integrity of the computed solution which is pivoting for automated automotive systems requiring guaranteed safety-critical solutions. Results from simulations and real experiments show that sharing position corrections reduces significantly satellite-specific GNSS errors effects in both cross-track and along-track components. Moreover, it is shown that lane-boundary measurements help reducing estimation errors for all the networked vehicles even those which are not equipped with an embedded perception system.
\end{abstract}

Keywords: ITS, Cooperative, Interval analysis, Bounded-error, GNSS and Sensor fusion. ${ }^{1}$

\section{Introduction}

Automated driving in city centres has the potential to reduce casualties and traffic jams (Alonso , 2011) -(Choi , 2015). Driving in high density traffic and different roadway infrastructures is a big challenge for automated vehicles and requires the vehicle's pose and speed to be accurately determined. Standard GNSS positioning (or standalone receiver positioning) is widely used in autonomous navigation but is not enough accurate, particularly in urban areas because of the reduced visibility of the satellites. GNSS positioning alone in constraints environments lacks of integrity even with multi-constellation receivers mixing GPS, Glonass or Galileo systems. A solution is to complement this technology with other absolute sensor measures from the vehicles in a data fusion approach. Indeed, a single sensor cannot provide the required level of performance, as autonomous vehicles need not only accurate positioning but also some guaranty on the quality of the computed solution. An new interesting solution is coming from V2X communication. Indeed, the data fusion of multiple sources between vehicles data enables a vehicle to determine its position more accurately and with more confidence as it merges its own sensor data with data shared by other vehicles.

Email addresses: conde@isr.uc.pt (Luís Conde Bento), philippe.bonnifait@hds.utc.fr (Philippe Bonnifait), urbano@isr.uc.pt (Urbano Nunes)

${ }^{1}$ Transportation Research Part C: Emerging Technologies, Volume 79, June 2017, Pages 42-57, ISSN 0968-090X, http://dx.doi.org/10.1016/j.trc.2017.01.002 
In this paper, we present a new absolute positioning algorithm called Lane Boundary Cooperative Augmented Set-membership GNSS Positioning LB-CASGP. This method uses shared GNSS corrections among connected vehicles (inter-vehicles) and fuses local information (intra-vehicle) from GNSS, digital maps and lane boundary detection. The solver relies on a bounded-error algorithm to improve estimation accuracy while keeping a high level of integrity. This approach guarantees with respect to a chosen integrity risk that the real vehicle position is included in the estimated set-membership domain, even if the equations are highly non-linear and even if the same information is reused several times in the computation.

The research described in this paper makes the following contributions to existing literature. The proposed $L B$-CASGP uses V2V/V2I communications to share GNSS differential corrections to all networked vehicles, when subjected to the same satellite-specific GNSS biases (ionosphere, troposphere, satellite clock). The algorithm generates differential corrections using data from connected vehicles, thus eliminating the need to have stationary receivers at known locations. The sharing of GNSS differential corrections for the position estimation, in a set-membership algorithm rather than in a classical iterative least square, is another contribution of this paper.

The $L B$-CASGP algorithm guarantees integrity by assuring that the position, obtained after applying the cooperative corrections, is inside a guaranteed risk integrity zone computed using a non-cooperative Lane Boundary Augmented Set-membership GNSS Positioning ( $L B-A S G P$ ). The zone computed using $L B-A S G P$ is constrained by geo-referenced lane boundary measurements and GNSS pseudoranges. The sensor fusion approach exploits lane boundary measurements to improve cross-track vehicle positioning. The along-track positioning error is also improved when using $L B-C A S G P$ and sharing cross-track errors among networked vehicles.

The algorithm only requires a small size database of the infrastructure road network as lane boundaries are described solely by "point-slope" data. The wireless communication bandwidth is quite small as the data to be transferred is only composed by two floating numbers per vehicle. Most of the existing data models used in absolute positioning studies consider often more complex databases and transmit full constellation layout and all satellites pseudoranges.

Simulations and real experiments were conducted to test the performance of the $L B-C A S G P$ algorithm, both in terms of accuracy and integrity. This algorithms has been implemented with low cost sensors and extensive comparative tests have been performed to evaluate its performance in comparison with the Set-membership GNSS Positioning algorithm (SGP) proposed in (Drevelle, 2009).

\section{Related Work}

Augmenting GNSS localization with other sensory information to improve the positioning accuracy is common in the context of intelligent vehicles (Toledo-Moreo , 2010)(Sun , 2015). In (Drawil , 2010), N.M. Drawil developed a V2V communication assisted localization. This localization technique takes advantage of the fact that GNSS receivers operating in close proximity and observing the same constellation of satellites have strongly correlated errors. These errors are largely canceled when a relative positioning system is taken into consideration. M. Woo, et al. (Woo , 2001) used V2V, GNSS and the distance among vehicles given by a vision and/or ranging sensor to compute a relative vehicle positioning. Although many current relative vehicle positioning methods are sufficient for platooning, their performance is not high enough to implement autonomous driving. GNSS accuracy is often enhanced by using carrier phase measurements (RTKGPS) and real-time corrections (Williams, 2012). G. Challitasing, et al. (Challita, 2009) used V2V communications, RTKGPS and a vehicle to vehicle ranging system (vision-based ranging system) for absolute positioning. Although this configuration is more performant, the RTKGPS system employed is expensive and therefore not available for massive vehicle distribution.

An algorithm to detect multi-lane marks, including driving lane marks and adjacent lane marks is presented in (Hur , 2013). This algorithm is able to detect multi-lane marks successfully in the absence of parallelism, thus enabling the algorithm to manage various non-parallel lane situations, such as are found at intersections, in splitting lanes, and in merging lanes. An approach taken for outdoor absolute positioning is presented in (Rife , 2010) and (Rife , 2012), where vehicles determine their positions in a cooperative way, by fusing their own sensor data with data shared by other users via a common communication network. These papers present cooperative 
navigation algorithms to increase the accuracy of vehicle positioning via the sensor information sharing through a V2V network. In (Rife , 2010) the algorithm generates GNSS differential corrections from a set of GNSS equipped vehicles by fusing GNSS measurements with a camera-based lane-boundary sensor. The results show that it is possible to generate an error-free differential correction that estimates the projection into the ground plane of the satellite-specific GNSS biases (ionosphere, troposphere, satellite clock) experienced by all collaborators in a local area. The benefits of the proposed method are more noticeable when the density of users is high. In (Rife , 2012), an algorithm for differential GNSS corrections with no stationary reference receiver is proposed. The algorithm generates differential corrections using data from moving vehicles, thus eliminating the need for an infrastructure of stationary receivers. This algorithm generates individual differential corrections for each satellite, shared among vehicles with different satellites in view. Results show that measurements sharing improves significantly the positioning accuracy in the cross-track and in the along-track direction.

The knowledge of localization uncertainties is of prime importance when the navigation of intelligent vehicles has to deal with safety issues. To quantify the localization confidence V. Drevelle et al. (Drevelle , 2009) (Drevelle , 2013) developed several algorithms based on interval analysis and constraint propagation. The developed algorithms can handle several hypotheses in cases of ambiguous solutions simply by computing disconnected solution sets and are able to compute location zones in which the user is guaranteed to be located. A set-membership based satellite positioning aided by height data from a digital elevation model (DEM) for high integrity was developed in (Drevelle, 2009). The integrity zone is computed recursively using a set-inversion method in a bounded-error context through set-bisection. Results show that the additional altitude information enabled more precise positioning while tolerating GPS outliers, especially with a small number of visible satellites. In (Drevelle , 2013), the algorithm has been enhanced with two stages. On a first stage, tightly coupled position domains are computed by constraint propagation on GNSS measurements and a precise 3D maps of the drivable space. A second stage provides localization integrity and information availability by the use of a position and proprioceptive data history. Results show that the algorithm is able to handle erroneous positions with a chosen integrity risk and, in the reported experiments carried out in urban canyons with bad satellite visibility, a full positioning availability has been obtained with errors smaller than $5.1 \mathrm{~m}$ during $95 \%$ of the trials.

\section{Theoretical Background}

\subsection{GNSS Pseudoranges}

A satellite-receiver pseudodistance, from satellite $s$ to receiver $r$, is obtained using binary sequence codes or carrier phase. Pseudorange measurements are affected by different physical phenomena that cause a delay in the propagation time of the signal. The main system error sources are: ionospheric $I_{r}^{s}$ and tropospheric $T_{r}^{s}$ biases along the signal path; satellite orbit estimation (or ephemeris) error $E^{s}$ and satellite clock offset $\delta t^{s}$. The most significant receiver dependent error source that affects also the pseudorange measurement is mainly linked to the accuracy of the receiver clock offset $\delta t_{r}$ estimate. $I_{r}^{s}$ is generated as the signal passes through the upper layer of the atmosphere. The gases therein are ionized by solar radiation, resulting in an increase of the propagation time of the signal. The error introduced can be up to $50 \mathrm{~m}$ for low elevation satellites. The tropospheric $T_{r}^{s}$ delay is mainly caused by water vapor, and it ranges from $2.5 \mathrm{~m}$ at the zenith to $15 \mathrm{~m}$ for low satellite elevations. All other error sources, such as relativistic errors, diffuse multipath and thermal noise are lumped in the $\epsilon_{r}^{s}$.

A pseudorange measurement based on a coarse/acquisition $(C / A)$ binary sequence code has typical errors at a meter scale and it is linked to the user position by the following equation (Kaplan , 1996):

$$
\rho_{r}^{s}=r_{r}^{s}+c \cdot\left(\delta t_{r}-\delta t^{s}\right)+I_{r}^{s}+T_{r}^{s}+E^{s}+\epsilon_{r}^{s}
$$

where $c$ is the speed of light in the vacuum.

The geometric range $r_{r}^{s}$ from receiver $r$ to satellite $s$ is given by:

$$
r_{r}^{s}=\sqrt{\left(x^{s}-x_{r}\right)^{2}+\left(y^{s}-y_{r}\right)^{2}+\left(z^{s}-z_{r}\right)^{2}}=\left\|\mathbf{x}^{\mathbf{s}}-\mathbf{x}_{\mathbf{r}}\right\|
$$




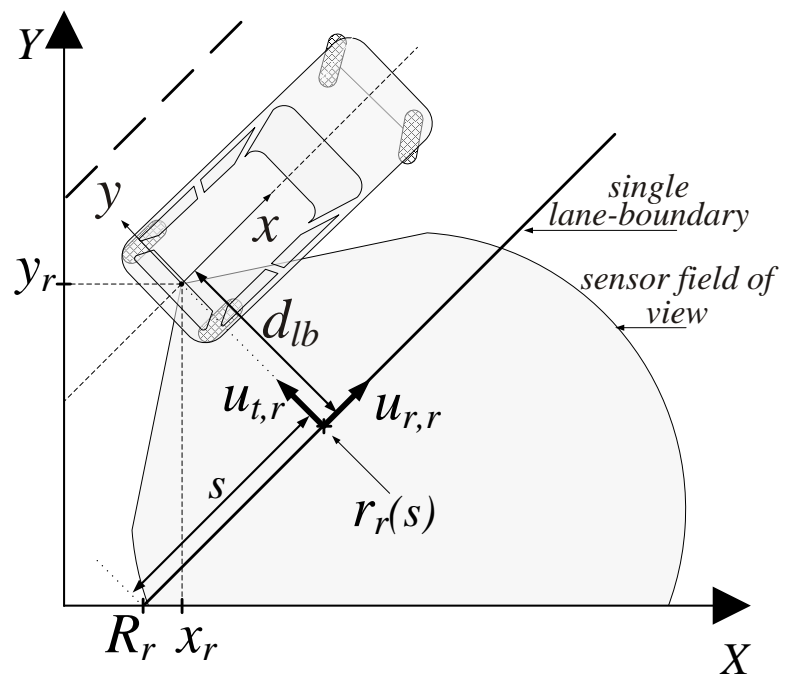

Figure 1: Lane-boundary distance measurement geometric layout

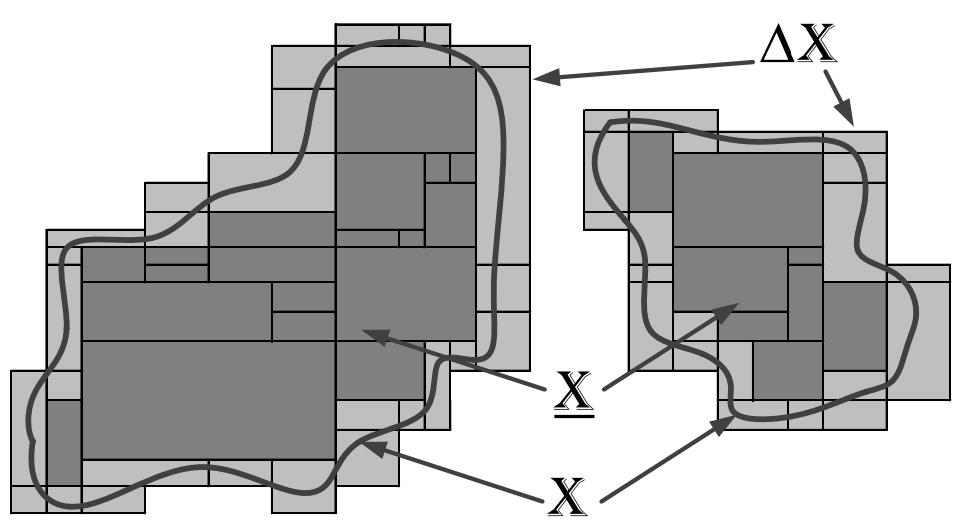

Figure 2: Compact set $\mathbb{X}$ : bracketed between an inner subpaving $\underline{\mathbb{X}}$ and an outer subpaving $\overline{\mathbb{X}}$, where $\overline{\mathbb{X}}$ is given by $\overline{\mathbb{X}}=\underline{\mathbb{X}}+\Delta \mathbb{X}$.

where $\mathbf{x}=(x, y, z) \cdot \mathbf{x}_{\mathbf{r}}$ and $\mathbf{x}^{\mathbf{s}}$ denote the position vectors of the receiver at the observation epoch (signal reception time) and of the satellite at the emission time, respectively. By converting the clock offset to distance units $c o_{r}$ and lumping all errors into $v_{r}^{s}$, one obtains:

$$
\rho_{r}^{s}=\left\|\mathbf{x}^{\mathbf{s}}-\mathbf{x}_{\mathbf{r}}\right\|+c o_{r}+v_{r}^{s}
$$

Using satellite positions and at least four measured pseudoranges, one can compute an estimate for both the receiver position $\mathbf{x}_{\mathbf{r}}$ and the receiver clock offset $c o_{r}$, via iterative nonlinear least squares (Kaplan , 1996).

\subsection{Lane Boundary Absolute Measurements}

Additional absolute measurements can be used to reduce the estimation error of the receiver position $\hat{\mathbf{x}}_{\mathbf{r}}$ and the receiver clock offset $\hat{c o}_{r}$. Geographic Information Systems (GIS) combined with lane-boundary sensors can provide additional absolute measurements. let us consider for simplification that the lane-boundary sensor is collocated with the GNSS receiver (see Figure 1).

Lane-boundary sensors only provide the distance to a line, i.e. the location along the lane boundary line is undefined. If the observed road is composed by a single straight line segment, the cross-track distance $d_{l b}$ to the lane boundary is unambiguous and can be expressed by:

$$
d_{l b}=\mathbf{u}_{\mathbf{t}, \mathbf{r}}^{T} \cdot\left(\mathbf{x}_{\mathbf{r}}-R_{r}\right)+\epsilon_{r}^{l b}
$$

where $R_{r}$ is the reference point of the lane frame $\left\{u_{t, r} ; u_{r, r}\right\}, \mathbf{u}_{\mathbf{t}, \mathbf{r}}$ is the cross-track unit vector and $\epsilon_{r}^{l b}$ models the lane-boundary sensor measurement errors.

\subsection{Set inversion}

Iterative least squares (Kaplan , 1996) are widely used for vehicle positioning. Set-membership methods solve the inversion problem in a different way. Interval analysis (Jaulin , 2001) involves intervals and their multidimensional extension, interval vectors (or boxes). A box is a subset of $\mathbb{R}^{n}$ defined as the Cartesian product of $n$ intervals $[\mathbf{x}]=[\overline{\mathbf{x}}, \underline{\mathbf{x}}]$. The set of real intervals is denoted $\mathbb{I R}$ and the set of $n$-dimensional boxes is $\operatorname{IR}^{n}$.

Let $\mathbf{f}: \mathbb{R}^{n} \rightarrow \mathbb{R}^{m}$ be a given function. The interval function $[\mathbf{f}]: \mathbb{I R}^{n} \rightarrow \mathbb{I R}^{m}$ is an inclusion function for $\mathbf{f}$ if

$$
\forall[\mathbf{x}] \in \mathbb{I I R}^{n}, \mathbf{f}([\mathbf{x}]) \subset[\mathbf{f}]([\mathbf{x}])
$$

To approximate compact sets in a guaranteed way, sub-pavings are used. A sub-paving of a box $[\mathbf{x}]$ is the union of nonempty and non-overlapping sub-boxes of $[\mathbf{x}]$. A guaranteed approximation of a compact set $\mathbb{X}$ can 
be made by bracketing it between an inner sub-paving $\underline{\mathbb{X}}$ and an outer sub-paving $\overline{\mathbb{X}}$ such as $\underline{\mathbb{X}} \subset \mathbb{X} \subset \overline{\mathbb{X}}$ (see Figure 2).

Given a known interval vector $\mathbb{Y}$ of $m$ measurements, the set inversion problem consists in determining the set $\mathbb{X}$, such that $\mathbf{f}(\mathbb{X}) \subset \mathbb{Y}$, where $\mathbb{Y} \subset \mathbb{R}^{m}$ (Tornil-Sin , 2010):

$$
\mathbb{X}=\mathbf{f}^{-1}(\mathbb{Y})=\left\{\mathbf{x} \in \mathbb{R}^{n} \mid \exists \mathbf{y} \in \mathbb{Y}, \mathbf{f}(\mathbf{x})=\mathbf{y}\right\}
$$

Given a arbitrarily large superset $\mathbb{X}_{0}$ where the solution set is known to belong an outer approximation $\overline{\mathbb{X}}$ can be computed using the Set Inversion Via Interval Analysis (SIVIA) algorithm (Jaulin , 1993). Feasible boxes are added to the inner sub-paving $\underline{\mathbb{X}}$ of solutions, a box $[\mathbf{x}]$ is feasible if $[\mathbf{f}]([\mathbf{x}]) \subset \mathbb{Y}$. Unfeasible boxes are discarded since they contain no solution. A box $[\mathbf{x}]$ is unfeasible if $[\mathbf{f}]([\mathbf{x}]) \cap \mathbb{Y}=\emptyset$. Indeterminate boxes are bisected into two sub-boxes waiting to be examined. A box $[\mathbf{x}]$ is indeterminate if $[\mathbf{f}]([\mathbf{x}])$ intersects but is not included in $\mathbb{Y}$. Algorithm termination is ensured by adding indeterminate boxes whose width is less than $\varepsilon$ to the sub-paving $\Delta \mathbb{X}$ of indeterminate boxes. Since we are seeking to characterize the positioning confidence domain, we only need to compute the outer sub-paving $\overline{\mathbb{X}}$ of the set that fulfills positioning constraints. Thus, the outer sub-paving is $\overline{\mathbb{X}}=\underline{\mathbb{X}}+\Delta \mathbb{X}$ (see Figure 2). Several special functions of interval computations are used throughout this paper, namely the midpoint or centre of an interval is given by $\operatorname{mid}(X)=\frac{\bar{X}+\underline{X}}{2}$, the width of an interval is defined as $\operatorname{wid}(X)=\bar{X}-\underline{X}$ and the magnitude of an interval is computed as follows $\operatorname{mag}(X)=\max (|\bar{X}|,|\underline{X}|)$.

\subsection{Measurement bounds setting}

If one wants to compute a confidence domain with a constant risk whatever the number of measurements, what is particular with bounded error methods is that the measurement bounds have to be chosen before every new computation. Indeed, these bounds are computed taking into account the global risk $\mathbf{R}$ and the number of tolerated spurious pseudoranges.

The probability of having at least $m-q$ good measurements is:

$$
\operatorname{Pr}\left(N_{o k} \geq m-q\right)=\sum_{i=m-q}^{m} \frac{m !}{i !(m-i) !} \cdot(1-r)^{i} \cdot(r)^{m-i}
$$

If there are no spurious pseudoranges (i.e. $q=0$ ), the SIVIA algorithm computes an outer approximation $\overline{\mathbb{X}}$ of the solution set $\mathbb{X}$ which is guaranteed to be consistent with the true position $x$. The maximum risk $r$ that can be assumed on each pseudorange interval, for a global risk $\mathbf{R}$, is given by:

$$
\begin{array}{ccc}
\operatorname{Pr}(x \in \overline{\mathbb{X}}) & \geq & \operatorname{Pr}\left(N_{o k} \geq m-q\right) \\
\operatorname{Pr}(x \notin \overline{\mathbb{X}}) & \leq & 1-\operatorname{Pr}\left(N_{o k} \geq m-q\right) \\
\mathbf{R} & \leq & 1-\sum_{i=m-q}^{m} \frac{m !}{i !(m-i) !} \cdot(1-r)^{i} \cdot(r)^{m-i}
\end{array}
$$

Once the maximum risk $r$ of each measurement interval to not contain the actual value is computed, the measurement error bounds can be set to meet this requirement. A centered Gaussian distribution with a variance $\sigma^{2}$ is used here as the error measurement model to set the error bounds on each pseudorange measurement $\rho_{r}^{s}$ :

$$
\left\{\begin{array}{l}
{\left[\rho_{r}^{s}\right]=\left[\rho_{r}^{s}-\alpha \sigma, \rho_{r}^{s}+\alpha \sigma\right]} \\
\alpha=-\Phi^{-1}\left(\frac{r}{2}\right)
\end{array}\right.
$$

where $\Phi$ is the cumulative distribution function of the standard normal distribution. This method leads to set the same amount of risk taken on each tail of the pseudorange Gaussian distribution.

\section{Position Estimation Algorithms}

The procedure involved in determining the receiver position consists in finding the set of positions compatible with the measurements and their associated bounded-error. Set-inversion methods (Jaulin, 2001) used to solve the problem of set-location do not add any risk, i.e. it is guaranteed not to lose solutions in the calculation. The risk that the interval does not contain the true position depends only on the risk that the selected intervals for the measures do not contain the true value. 


\subsection{Set membership GNSS Positioning}

The Set membership GNSS Positioning (SGP) method (Drevelle , 2009) consists in finding a location zone given uncertainty on satellites observations and satellites positions. GNSS absolute positioning requires satellites observations $\rho_{r}^{s}$ as well as their positions $\mathbf{x}^{\mathbf{s}}=\left(x^{s}, y^{s}, z^{s}\right)$ at the time of transmission. Satellite positions are known with uncertainty. Therefore, for set-membership positioning, each satellite position is represented as a box $\left[\mathbf{x}^{\mathbf{s}}\right]=\left(\left[x^{s}\right],\left[y^{s}\right],\left[z^{s}\right]\right)$ whose bounds are chosen to contain the true satellite position at a given confidence level. As mentioned in Section 3.1, pseudorange measurements being inaccurate for set-membership positioning, so pseudorange measurements are modelled as intervals $\left[\rho_{r}^{s}\right]$ whose bounds are determined given a chosen risk.

As there is a receiver clock offset, GNSS positioning is a four-dimensional problem and at least four satellites observations are necessary to estimate the GNSS receiver position. The GNSS receiver position zone computation consists in finding the set $\mathbb{X}$ of all locations compatible with the $m$ available measurements and the corresponding satellite positions which are also manipulated as boxes:

$$
\begin{aligned}
\mathbb{X}_{S G P}= & \left\{\left(x_{r}, y_{r}, z_{r}, c o_{r}\right) \in \mathbb{R}^{4} \mid \forall s=1 \cdots m, \exists \rho_{r}^{s} \in\left[\rho_{r}^{s}\right],\right. \\
& \left.\exists\left(x^{s}, y^{s}, z^{s}\right) \in\left[\mathbf{x}^{\mathbf{s}}\right], \rho_{r}^{s}=\left\|\mathbf{x}^{\mathbf{s}}-\mathbf{x}_{\mathbf{r}}\right\|+c o_{r}\right\} \\
\mathbb{Y}_{S G P}= & \left(\left[\rho_{r}^{s}\right],\left[\mathbf{x}^{\mathbf{s}}\right]\right)
\end{aligned}
$$

The subpaving $\mathbb{X}$ has a dual nature. It may be seen as a subset of $\mathbb{R}^{4}$ and it also can be viewed as a finite list of boxes $[X]$ (Jaulin , 2001). In order to be useful, for instance to a path following controller, a punctual 2D position vector has to be estimated. The resulting set is not only composed of $3 \mathrm{D}$ boxes as it includes the clock offset $c_{r}$, making it a $4 \mathrm{D}$ set. Boxes in the subpaving do not have all the same size (see Figure 2). Therefore, a solution to provide punctual estimation with the $4 \mathrm{D}$ set is to compute for each of the 4 dimensions, the mean of the geometric center of all boxes weighted by the volume of each box. In a single-frequency GNSS navigation solution with raw observations, the $c o_{r}$ is dominant over atmospheric residuals and noise. A more accurate solution of the $3 \mathrm{D}$ punctual location estimate is therefore to compute the $3 \mathrm{D}$ the mean of the geometric center of all boxes weighted by two parameters. First, the contribution of a box to the final solution is weighted by its width Second, the weight is also a function of the $c o_{r}$ of each box. The weight of a box to the final solution decreases as its punctual estimate of the clock offset is far from the weighted average $\hat{c o}{ }_{r}$.

Given $\left([X]=\left(\left[x_{r}\right],\left[y_{r}\right],\left[z_{r}\right],\left[c o_{r}\right]\right)\right)$, a punctual estimate of $\hat{c o}$ can be computed by using the center of gravity of the $X(4)$ component, for all $n$ boxes:

$$
\hat{c} o_{r}=\frac{\sum_{k=1}^{n}\left(\operatorname{mid}\left(X_{k}(4)\right) \cdot \operatorname{wid}\left(X_{k}(4)\right)\right)}{\sum_{k=1}^{n} \operatorname{wid}\left(X_{k}(4)\right)} \mid \forall\left[X_{k}(4)\right] \in \mathbb{X} \wedge n=\# \mathbb{X}
$$

where $\# \mathbb{X}$ is the number of boxes belonging to the subset and $\sum_{k=1}^{n} \mathbf{w i d}\left(X_{k}(4)\right)$ is the normalisation term.

The estimated 3D position vector $\hat{\mathbf{x}}_{\mathbf{r}}=\left(\hat{x}_{r}, \hat{y}_{r}, \hat{z}_{r}\right)$ is obtained by computing the center of gravity of the sub-paving, weighted by the value of the estimated receiver clock offset $\hat{c} o_{r}$ of each sub-paving:

$$
\hat{\mathbf{x}}_{\mathbf{r}}=\frac{\sum_{k=1}^{n}\left(\operatorname{mid}\left(X_{k}(i)\right) \cdot \operatorname{wid}\left(X_{k}(i)\right) \cdot C_{w f}(k)\right)}{\sum_{p=1}^{n} \operatorname{wid}\left(X_{p}(i)\right)} \mid \forall\left[X_{k}(i)\right] \in \mathbb{X} \wedge n=\# \mathbb{X} \wedge i=1, \cdots, 3
$$

where $\sum_{p=1}^{n} \operatorname{wid}\left(X_{p}(i)\right)$ is the sum of all boxes lengths along $i$ axis and $C_{w f}$ is the $\hat{c o} r$ weighting factor:

$$
C_{w f}(k)=\frac{1-\frac{\| \operatorname{mid}\left(X_{k}(4)\right)\left|-\hat{c} o_{r}\right|}{\left|\operatorname{mag}(X(4))-\hat{c} o_{r}\right|}}{\sum_{j=1}^{n}\left(1-\frac{\| \operatorname{mid}\left(X_{j}(4)\right)\left|-\hat{c o} r_{r}\right|}{\left|\operatorname{mag}(X(4))-\hat{c} o_{r}\right|}\right)} \mid \forall\left[X_{k}(4)\right] \in \mathbb{X} \wedge n=\# \mathbb{X}
$$

The final $2 \mathrm{D}$ position $\left[x_{r}, y_{r}\right]$ estimate is determined by projecting onto a flat plane the $3 \mathrm{D}$ solution $\hat{\mathbf{x}}_{\mathbf{r}}$. 


\subsection{Lane Boundary Cooperative Augmented set membership GNSS Positioning (LB-CASGP)}

Lane-boundary measurements can provide corrections to improve the position estimate of a single receiver using $L B$-ASGP, but an improvement by means of a cooperative vehicle positioning (LB-CASGP) can be achieved by sharing this corrections among vehicles.

With the assumption that the model and the measurement errors are bounded, both GNSS pseudoranges and lane-boundary data can be fused by using a set-inversion approach in such a way that all the results are guaranteed (Drevelle , 2009). Given the road network information provided by a GIS database, a constraint represented by the cross-track vector can be applied to a box $[\mathbf{x}]$. To apply this constraint, the road network information is first transformed into the coordinate system used in the GNSS, i.e. convert from local-leveltangent ENU (East North Up) coordinates to WGS-84 (World Geodetic System 1984) ECEF (Earth-Centered, Earth-Fixed) Cartesian coordinates.

The cross-track vector is represented as a box $\left[\mathbf{u}_{\mathbf{t}, \mathbf{r}}\right]$ whose bounds are chosen to contain the true cross-track vector. The measurement inaccuracy of the perpendicular distance given by the lane-boundary sensor with respect to the lane boundary is modelled as an interval $\left[d_{l b}\right]$ whose bounds are determined according to the lane-boundary sensor characteristics. Intervals are used to express the uncertainties of the information stored in the GIS database and measurement inaccuracies of the lane-boundary sensor.

The search area is expanded on the horizontal plane defined by the road segment. The set-membership GNSS positioning location set $\mathbb{X}$ is then reduced after contraction which removes every location area not compatible with the lane-boundary sensor measurements:

$$
\begin{aligned}
& \mathbb{X}_{L B-A S G P}=\left\{\left(x_{r}, y_{r}, z_{r}, c o_{r}\right) \in \mathbb{R}^{4} \mid \forall s=1 \cdots m, \exists \rho_{r}^{s} \in\left[\rho_{r}^{s}\right],\right. \\
& \exists\left(x^{s}, y^{s}, z^{s}\right) \in\left[\mathbf{x}^{\mathbf{s}}\right], \exists \mathbf{u}_{\mathbf{t}, \mathbf{r}} \in\left[\mathbf{u}_{\mathbf{t}, \mathbf{r}}\right], \exists d_{l b} \in\left[d_{l b}\right], \\
& \left.\mid \begin{array}{l}
\rho_{r}^{s}=\left\|\mathbf{x}^{\mathbf{s}}-\mathbf{x}_{\mathbf{r}}\right\|+c o_{r} \\
d_{l b}=\mathbf{u}_{\mathbf{t}, \mathbf{r}}{ }^{T} \cdot\left(\mathbf{x}_{\mathbf{r}}-R_{r}\right)
\end{array}\right\} \\
& \mathbb{Y}_{L B-A S G P}=\left(\left[\rho_{r}^{s}\right],\left[\mathbf{x}^{\mathbf{s}}\right],\left[\mathbf{u}_{\mathbf{t}, \mathbf{r}}\right],\left[d_{l b}\right]\right)
\end{aligned}
$$

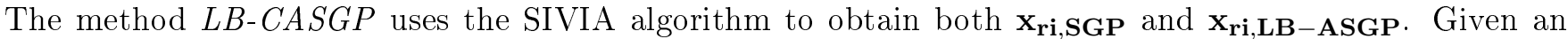
arbitrarily large superset $\mathbb{X}_{0}$ where the solution set is known to belong, an outer approximation $\overline{\mathbb{X}}$ is computed. A box $[\mathbf{x}]$ is added to the inner subpaving $\underline{\mathbb{X}}$ of solutions if $[\mathbf{f}]([\mathbf{x}]) \subset \mathbb{Y}$.

Algorithms 1 and $\mathbf{2}$ resumes the proposed method $(L B-C A S G P)$, where the functions $\mathbf{f}$ to invert are given by:

$$
\mathbf{f}_{\mathbf{S G P}}=\left\{\rho_{r}^{s}=\left\|\mathbf{x}^{\mathbf{s}}-\mathbf{x}_{\mathbf{r}}\right\|+c o_{r}\right.
$$

and

$$
\mathbf{f}_{\mathbf{L B}-\mathbf{A S G P}}=\left\{\begin{array}{l}
\rho_{r}^{s}=\left\|\mathbf{x}^{\mathbf{s}}-\mathbf{x}_{\mathbf{r}}\right\|+c o_{r} \\
d_{l b}=\mathbf{u}_{\mathbf{t}, \mathbf{r}}^{T} \cdot\left(\mathbf{x}_{\mathbf{r}}-R_{r}\right)
\end{array}\right.
$$

As observed in Figure 3, the LB-CASGP (Algorithms 1 and 2) solves simultaneously three problems, two set inversion problems (LB-ASGP and SGP) and the satellite specific corrections (ssc) $\left[C_{s s c}\right]=\left[C_{N, s s c} ; C_{E, s s c}\right]$.

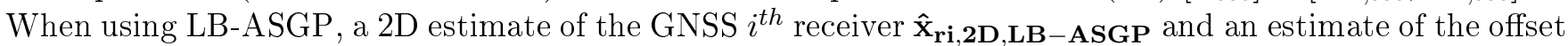
along the lateral direction of the road $C_{i, L B-A S G P}$ are obtained. From an initial searching volume $\mathbb{X}_{0}$, it is first found the set $\mathbb{X}_{S G P}$ of all locations compatible with the measurements $\left[\rho_{r}^{s}\right]$ and the satellite position intervals $\left[\mathbf{x}^{\mathbf{s}}\right]$ using function $\mathbf{f}_{\mathbf{S G P}}$. Starting with the $\mathbb{X}_{S G P}$ computed previously and using function $\mathbf{f}_{\mathbf{L B}-\mathbf{A S G P}}$, it is computed the set $\mathbb{X}_{L B-A S G P}$ of all locations compatible with the measurements $\left[\rho_{r}^{s}\right]$, the satellite position intervals 


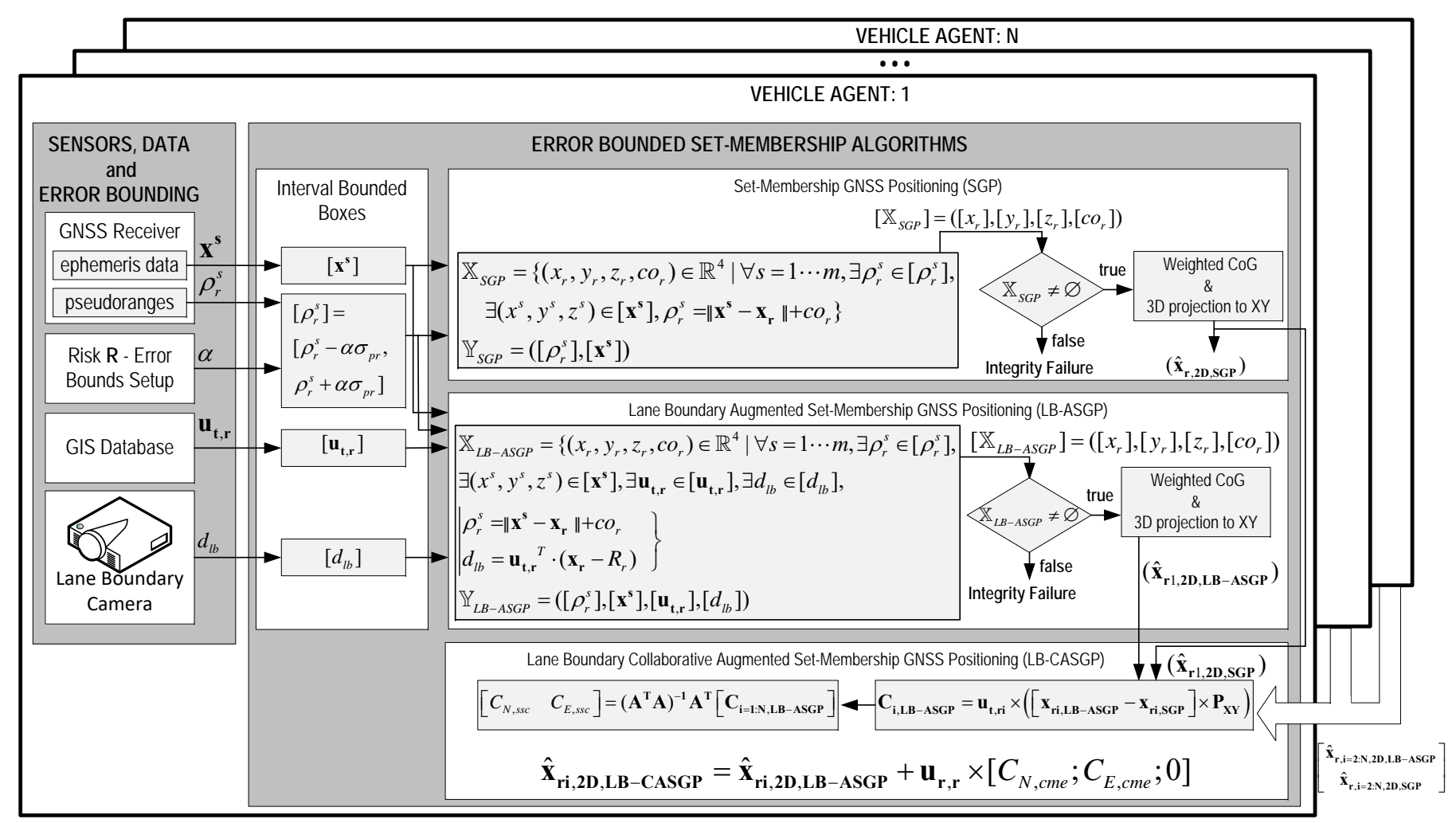

Figure 3: Dataflow and LB-CASGP algorithm architecture.

$\left[\mathbf{x}^{\mathbf{s}}\right]$, the cross-track vector $\left[\mathbf{u}_{\mathbf{t}, \mathbf{r}}\right]$ and the lane boundary $\left[d_{l b}\right]$.

$$
\begin{aligned}
& \text { Algorithm 1: }\left[\hat{\mathbf{x}}_{\mathbf{r}, \mathbf{2 D}, \mathbf{L B}-\mathbf{C A S G P}} ; \mathbf{C}_{\mathbf{i}, \mathbf{L B}-\mathbf{A S G P}]}\right]=C A S G P \_L B \\
& \left(\left[\mathbf{f}_{\mathbf{S G P}}\right],\left[\mathbf{f}_{\mathbf{L B}-\mathbf{A S G P}}\right], \mathbb{Y}_{S G P}, \mathbb{Y}_{L B-A S G P}, \mathbb{X}_{0}, \mathbf{C}_{\mathbf{n e t}, \mathbf{L B}-\mathbf{A S G P}}\right) \\
& \mathfrak{L} \leftarrow \operatorname{root}\left(\mathbb{X}_{0}\right) \\
& \hat{\mathbf{x}}_{\mathbf{r}}(\mathbf{1})=P P E\left(\left[\mathbf{f}_{\mathbf{S G P}}\right], \mathbb{Y}_{S G P}, \mathfrak{L}\right) \\
& \hat{\mathbf{x}}_{\mathbf{r i}, \mathbf{2 D}, \mathbf{S G P}}=\left[\hat{x}_{r, 2 D}, \hat{y}_{r, 2 D}\right]=\hat{\mathbf{x}}_{\mathbf{r}}(1) \times \mathbf{P}_{\mathbf{X Y}} \\
& \text { if WithLaneSensor then } \\
& \hat{\mathbf{x}}_{\mathbf{r}}(\mathbf{2})=P P E\left(\left[\mathbf{f}_{\mathbf{L B}-\mathbf{A S G P}}\right], \mathbb{Y}_{L B-A S G P}, \mathfrak{L}\right) \\
& \hat{\mathbf{x}}_{\mathbf{r i}, 2 \mathrm{D}, \mathbf{L B}-\mathbf{A S G P}}=\left[\hat{x}_{r, 2 D}, \hat{y}_{r, 2 D}\right]=\hat{\mathbf{x}}_{\mathbf{r}}(2) \times \mathbf{P}_{\mathbf{X Y}} \\
& \mathbf{C}_{\mathbf{i}, \mathbf{L B}-\mathbf{A S G P}}=\mathbf{u}_{\mathbf{t}, \mathbf{r i}} \times\left(\left[\hat{\mathbf{x}}_{\mathbf{r}}(2)-\hat{\mathbf{x}}_{\mathbf{r}}(1)\right] \times \mathbf{P}_{\mathbf{X Y}}\right) \\
& \text { end if } \\
& {\left[C_{s s c}\right]=\left[C_{N, s s c} ; C_{N, s s c}\right] \leftarrow \text { equation (18) }} \\
& \text { if WithLaneSensor then } \\
& \hat{\mathbf{x}}_{\mathbf{r i}, 2 \mathrm{D}, \mathbf{L B}-\mathrm{CASGP}}=\hat{\mathbf{x}}_{\mathbf{r i}, 2 \mathrm{D}, \mathbf{L B}-\mathbf{A S G P}}+\mathbf{u}_{\mathrm{r}, \mathrm{r}} \times \\
& {\left[C_{N, s s c} ; C_{E, s s c} ; 0\right]} \\
& \text { else } \\
& \mathbf{x}_{\mathbf{r i}, 2 \mathbf{D}, \mathbf{L B}-\mathbf{C A S G P}}=\mathbf{x}_{\mathbf{r i}, \mathbf{2 D}, \mathbf{S G P}}+\left[C_{N, s s c} ; C_{E, s s c} ; 0\right]
\end{aligned}
$$

Using sets $\mathbb{X}_{S G P}$ and $\mathbb{X}_{L B-A S G P}$, the $3 \mathrm{D}$ position estimate of the GNSS $i^{\text {th }}$ receiver $\hat{\mathbf{x}}_{\text {ri,LB-ASGP }}$ is obtained by computing the center of gravity of the sub-paving, weighted by the value of the estimated receiver

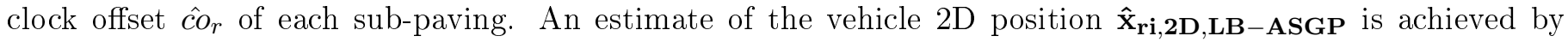

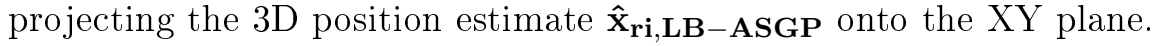

Lane-boundary constraints can further improve the performance of the set-membership GNSS location zone determination, if satellite-specific errors estimations obtained using lane-boundary are share among connected vehicles. $L B-C A S G P$ is a decentralized algorithm and it uses cross-track corrections shared among networked vehicles in order two improve the along-track vehicle position.

The component of the satellite-specific errors which causes the $i^{\text {th }}$ receiver position estimate to suffer 


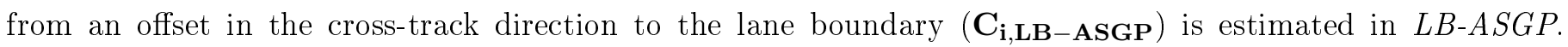
The $\mathbf{C}_{\mathbf{i}, \mathbf{L B}-\mathbf{A S G P}}$ is obtained by taking into account only the cross-track component that has shifted the estimated position computed using the $S G P$ algorithm to the estimated position computed using $L B$-ASGP. Each vehicle equipped with a lane-boundary sensor shares its correction to the networked vehicles. Given the position estimates of vehicle $i$ given by the SGP algorithm $\hat{\mathbf{x}}_{\mathbf{r i}, \mathbf{S G P}}=\left[\hat{x}_{r i, S G P}, \hat{y}_{r i, S G P}, \hat{z}_{r i, S G P}\right]$ and by the $L B$ $A S G P$ algorithm $\hat{\mathbf{x}}_{\mathbf{r i}, \mathbf{L B}-\mathbf{A S G P}}=\left[\hat{x}_{r i, L B-A S G P}, \hat{y}_{r i, L B-A S G P}, \hat{z}_{r i, L B-A S G P}\right]$, the cross-track component error of a single vehicle is obtained by differencing both estimates and computing the vector along the cross-track direction:

$$
\mathbf{C}_{\mathbf{i}, \mathbf{L B}-\mathrm{ASGP}}=\mathbf{u}_{\mathrm{t}, \mathrm{ri}} \times\left(\left[\mathrm{x}_{\mathrm{ri}, \mathrm{LB}-\mathrm{ASGP}}-\mathrm{x}_{\mathrm{ri}, \mathrm{SGP}}\right] \times \mathbf{P}_{\mathrm{XY}}\right)
$$

where $i=1 \ldots n$ for $n$ lane-boundary equipped vehicles.

The cooperative North (global Y-axis coordinates) and East (global X-axis coordinates) correction estimation w.r.t. to global coordinates, are respectively given by $C_{N, s s c}$ and $C_{E, s s c}$. They are computed using the

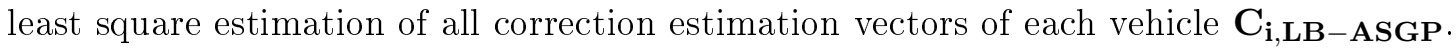

$$
\left[\begin{array}{c}
C_{N, s s c} \\
C_{E, s s c}
\end{array}\right]=\left(\mathbf{A}^{\mathbf{T}} \mathbf{A}\right)^{-\mathbf{1}} \mathbf{A}^{\mathbf{T}}\left[\begin{array}{c}
\mathbf{C}_{\mathbf{1}, \mathbf{L B}-\mathbf{A S G P}} \\
\vdots \\
\mathbf{C}_{\mathbf{n}, \mathbf{L B}-\mathbf{A S G P}}
\end{array}\right]
$$

where $\mathbf{P}_{\mathbf{X Y}}$ is the projection matrix onto the $X Y$ plane, and $\mathbf{A}$ is given by:

$$
\mathbf{A}=\left[\begin{array}{c}
\mathbf{u}_{\mathbf{t}, \mathbf{r} \mathbf{1}} \\
\vdots \\
\mathbf{u}_{\mathbf{t}, \mathbf{r n}}
\end{array}\right]
$$

It was chosen to compute a punctual estimate of $\left[C_{s s c}\right]$ by using least squares to avoid transferring between vehicles large amounts of data, i.e. avoid the broadcast of all boxes belonging to the solution set of each vehicle using SGP and LB-ASGP. This decentralized solution has a minimal data transfer as it only requires the reception of $\mathbf{C}_{\mathbf{i}, \mathbf{L B}-\mathbf{A S G P}}$ and $\mathbf{u}_{\mathbf{t}, \mathbf{r i}}$ from the vehicles equipped with lane-boundary sensors. Those vehicles have a good cross-track accuracy and a poor one in the along-track direction. Therefore, position estimates using $L B$-CASGP algorithm for vehicles equipped lane-boundary sensor, are only corrected (w.r.t. LB-ASGP) along the along-track component $\mathbf{u}_{\mathbf{r}, \mathbf{r}}$ using the shared corrections:

$$
\begin{aligned}
& \hat{\mathbf{x}}_{\mathbf{r i}, 2 \mathbf{D}, \mathbf{L B}-\mathbf{C A S G P}}=\hat{\mathbf{x}}_{\mathbf{r i}, 2 \mathrm{D}, \mathbf{L B}-\mathbf{A S G P}+} \\
& \mathbf{u}_{\mathbf{r}, \mathbf{r}} \times\left[C_{N, s s c} ; C_{E, s s c} ; 0\right]
\end{aligned}
$$

For vehicles not equipped with lane-boundary sensors, the shared corrections are applied in both cross-track and along-track components:

$$
\hat{\mathbf{x}}_{\mathbf{r i}, 2 \mathbf{D}, \mathbf{L B}-\mathbf{C A S G P}}=\hat{\mathbf{x}}_{\mathbf{r i}, 2 \mathbf{D}, \mathbf{S G P}-\mathbf{L B}}+\left[C_{N, s s c} ; C_{E, s s c} ; 0\right]
$$

When all vehicles are collinear no solution can be obtained since there is a singularity. In this situation,

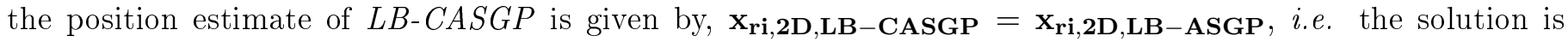
standalone without any cooperative information.

\section{Results}

To analyze the performance of the proposed set-membership positioning algorithm, simulations and real experiments are reported. Figure 9 shows the trajectory followed by all vehicles and position estimation for all presented algorithms, tested in simulation (Figure 9(a)) and in real experiments (Figure 9(b)). 


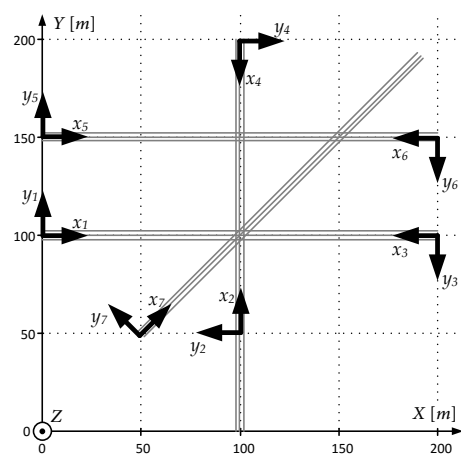

(a)
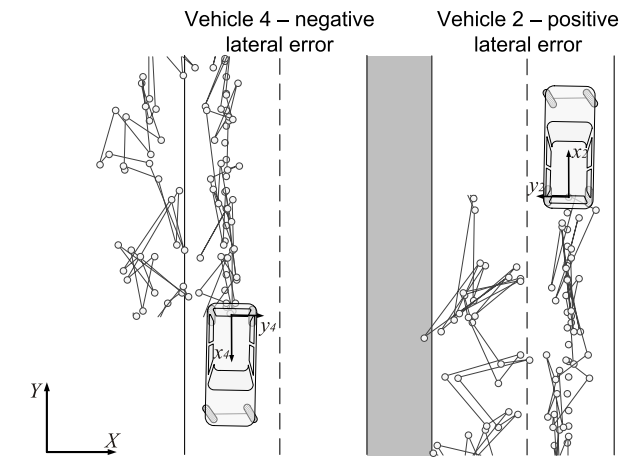

(b)

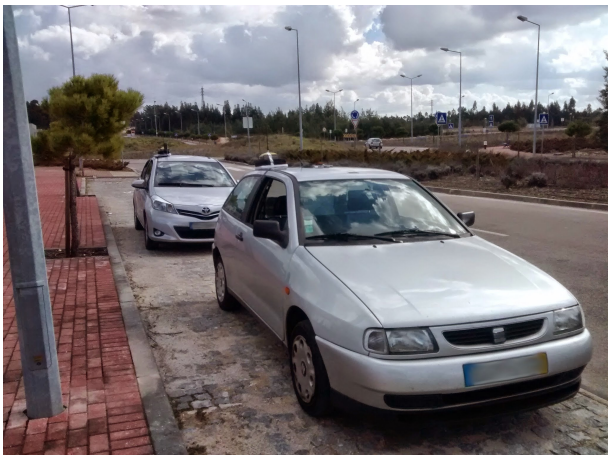

(c)

Figure 4: Setup: (a) Simulation environment - 7 vehicles simulated road network with starting vehicle positions and headings, vehicle $\mathrm{x}$-axis and $\mathrm{y}$-axis represent along-track and cross-track directions respectively; (b) Simulation - Zoomed trajectory and position estimation for vehicles 2 and 4 . Vehicles 2 and 4 travel in a road lane parallel to the $Y$-axis local coordinates system $\Sigma$. Vehicle 2 is rotated by $\pi / 2$ with respect to $\Sigma$. Vehicle 4 is rotated by $3 \pi / 2$ with respect to $\Sigma$. The two vehicle's $y$-axis have opposite directions, leading to a positive lateral error for vehicle 2 and a negative lateral error for vehicle 4 in each vehicle local frame. This satellite constellation configuration generates an error with the major axis along the negative direction of the $\Sigma X$-axis; (c) Real Experiment - instrumented vehicles used in the tests;

\subsection{Simulation Setup}

ISR-TRAFSIM 4.0 has been used as the simulation environment (available at:

http://www.isr.uc.pt/ conde/isr-trafsim/). It is an open-source Matlab-based simulator and it has been used in different studies such as automatic traffic control, vehicle emissions analysis (Bento , 2013), vehicle pathfollowing control (Nunes , 2007) and sensor fusion for vehicle awareness. Seven vehicles traveling on an urban road network have been simulated (see Figure 4(a)(c)). Each vehicle is marked with a pair of coordinate axes indicating local along-track and cross-track directions. All vehicles are equipped with V2V communications, but only vehicles 1-6 are equipped with lane-boundary sensor.

The GPSoft (Tetewsky , 1998) software was used to emulate the GNSS system, namely the USA GPS constellation (Kaplan , 1996). The GPSoft Toolbox emulates not only satellites and receivers but also the propagation channels. Error sources such as thermal noise, multipath, atmospheric delays and Selective Availability are modelled as an integral part of pseudorange and integrated Doppler emulation. Furthermore, the errors are emulated such that the proper temporal and spatial correlation effects are observed in the measurements. This allows for realistic modelling of both code DGPS and carrier-phase RTKGPS in addition to usual stand-alone positioning algorithms. GPSoft also enables emulation of Galileo, GEOs, GPS and GPS Modernization (C/A-code on L1, L2 and L5) as well as dual-frequency P-code measurements. The user can emulate signals on additional carrier frequencies defined by the user. The satellite constellation emulator supports GPS and Glonass as well as user-defined constellations. In addition, YUMA-format broadcast almanacs can be used. The emulation of $\mathrm{C} / \mathrm{A}$ and $\mathrm{P}$-code pseudorange and integrated Doppler with user definable civil and military carrier frequencies is available including characteristics such as thermal noise, ionospheric delay, tropospheric delay and diffuse multipath (Tetewsky, 1998).

In this paper, the satellite-receiver distance from the satellite $s$ to the receiver $r$ is measured using $C / A-L 1$ code. As mentioned earlier, GNSS pseudoranges are affected by several types of error. The daily behavior of the ionospheric delay has been emulated using a half cosine function of the local time during daytime and by a constant level during nighttime, scaled by a satellite elevation factor. The average ionospheric injected error is 4 meters. No scintillation events have been introduced and the daytime total electron content is bounded by $\left[4 \times 10^{17} ; 1.6 \times 10^{18}\right]$. The emulated tropospheric delay ranges from 3 meters for a satellite at zenith to 25 meters for a satellite at 5 degrees elevation. A white noise is passed through a first-order Butterworth low-pass filter to generate the code diffuse multipath error of zero-elevation angle, which is scaled by the cosine of the true satellite elevation angle before it is applied to the range measurement. The standard deviation of pseudorange diffuse multipath errors at zero-elevation is 1.6 meters. A different uncorrelated diffuse multipath error is generated for each satellite and receiver (Kaplan , 1996). The standard deviation of the thermal noise is 1 meter. 


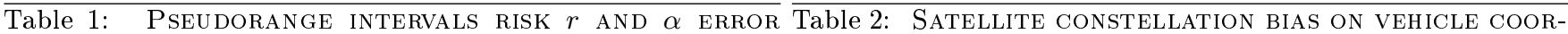
BOUNDS AS A FUNCTION OF THE NUMBER $m$ OF MEASURE- DiNATES AND VEHICle ORIENTATION MENTS

\begin{tabular}{c|cccc}
\hline \hline$m$ & 4 & 5 & 6 & 7 \\
\hline$r$ & $0.25 \cdot 10^{-4}$ & $0.20 \cdot 10^{-4}$ & $0.17 \cdot 10^{-4}$ & $0.14 \cdot 10^{-4}$ \\
$\alpha$ & 4.21 & 4.26 & 4.31 & 4.34 \\
\hline \hline$m$ & 8 & 9 & 10 & 11 \\
\hline$r$ & $0.13 \cdot 10^{-4}$ & $0.11 \cdot 10^{-4}$ & $0.10 \cdot 10^{-4}$ & $0.09 \cdot 10^{-4}$ \\
$\alpha$ & 4.37 & 4.39 & 4.42 & 4.44
\end{tabular}

\begin{tabular}{l|ccccccc}
\hline \hline Vehicle & \multicolumn{7}{|c}{ Vehicles } \\
Coordinates & 1 & 2 & 3 & 4 & 5 & 6 & 7 \\
\hline \multirow{2}{*}{-axis } & -- & + & ++ & - & -- & ++ & - \\
& - & & + & & - & + & \\
\hline \multirow{2}{*}{$Y$-axis } & + & ++ & - & -- & + & - & ++ \\
& & + & & - & & & + \\
\hline$\theta_{r}$ & 0 & $\pi / 2$ & $\pi$ & $3 \pi / 2$ & 0 & $\pi$ & $\pi / 4$
\end{tabular}

For a global risk $\mathbf{R}=10^{-4}$ and given the current number $m$ of measurements, the pseudorange intervals risk $r$ and the error bounds $\alpha$ are computed thanks to equations (Drevelle, 2009) and (Drevelle, 2013). The intervals $\left[\rho_{r}^{s}\right]=\left[\rho_{r}^{s}-\alpha \sigma, \rho_{r}^{s}+\alpha \sigma\right]$ are given in Table 1 for up to 11 satellites. The lane-boundary sensor error was assumed to have a standard deviation of $\sigma_{l b}=0.25 \mathrm{~m}$ and the lane-boundary interval was set to contain $95 \%$ of the measurements, i.e. $2 \times \sigma_{l b}$.

The satellite constellation setup produced a bias in the negative direction of the local frame $X$-axis (denoted by ---$)$ and a very small bias in the positive direction of the $Y$-axis (denoted by + ). In other words, this satellites constellation configuration generates an error with the major axis along the negative direction of the local coordinates $X$-axis (see Figure $4(\mathrm{c})$ ).

Table 2 presents the a qualitative evaluation of the satellite constellation setup bias on each vehicle coordinates, e.g. vehicle 1 axis are aligned with local coordinates, therefore the satellite constellation setup bias on vehicle 1 coordinates has the same direction and signal as local coordinates.

\subsection{Experimental Setup}

Standard road vehicles equipped with the same experimental setup have been used on real tests (see Figure 4(b)). The used test site allows to define a huge variety of paths. The surrounding environment is made of trees and buildings. Four ublox LEA-6T have been used as embedded GPS-receivers. This kind of receiver enables easy vehicle integration, have a standard communication interface and provides raw-data which are necessary for the pseudoranges processing. The ground-truth setup was a high performance RTKGPS system TOPCON HiperPro, able to provide positioning solutions with centimeter accuracy. The master antenna fixed station (MAFS) used by the RTK-GPS used data obtained through a SERVIR project facility which consists on a military network of permanent reference GNSS stations capable of providing raw-data observations and corrections for real-time RTK or post-processed PPK. During the experiments, one of these stations (SERVIR - Station 9) gathered all the necessary conditions to be used as MAFS: short distance to the test site, no multipath or electromagnetic interference sources nearby and no signal obstruction caused by trees or higher buildings. The lane-boundary measurements were obtained using the RTK-GPS with a $25 \mathrm{~cm}$ additional Gaussian error. Therefore, the global accuracy of the lane boundary measurement is in the order of the accuracy of a classical lane detection camera (Gat, 2005).

\subsection{Set-membership GNSS positioning (SGP)}

When using a set-membership GNSS method, an important focus is on the characterization of domains which contain the solution rather than the search of a punctual result which might be misleading and with no associated confidence information. In this work, the unknown variables are $\left(x_{r}, y_{r}, z_{r}, c o_{r}\right)$. The initial searching volume was set to $27 \times 10^{6}\left[\mathrm{~m}^{3}\right]$ which is an arbitrarily high value with little impact on the processing time.

Figures 5 and 6 present results of the distributions of the lateral (a) and longitudinal (b) error, for the simulation and the real experiments respectively.

From the simulation results depicted in Figure 5, it is possible to observe the bias along both $X$-axis and $Y$-axis local coordinates. The negative bias along the local coordinates $X$-axis (i.e. this satellite constellation configuration generates along local coordinates $X$-axis a predominant negative error) can be easily seen through $S G P$ lateral error of vehicles 2 and 4 and $S G P$ longitudinal error of vehicles $1,3,5$ and 6 . The simulated vehicles 2 and 4 travel in a road lane parallel to the local coordinates $Y$-axis (see Figure 4(a)). 

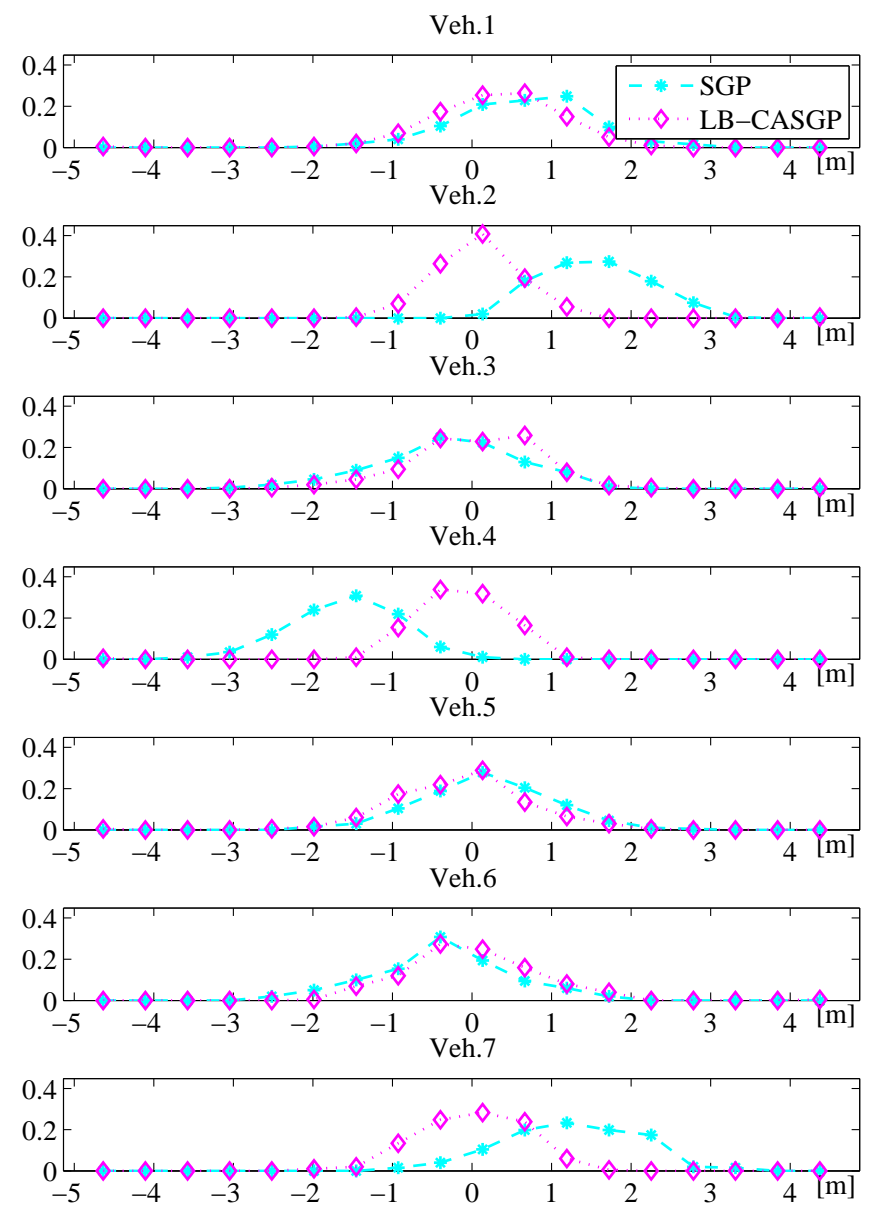

(a)
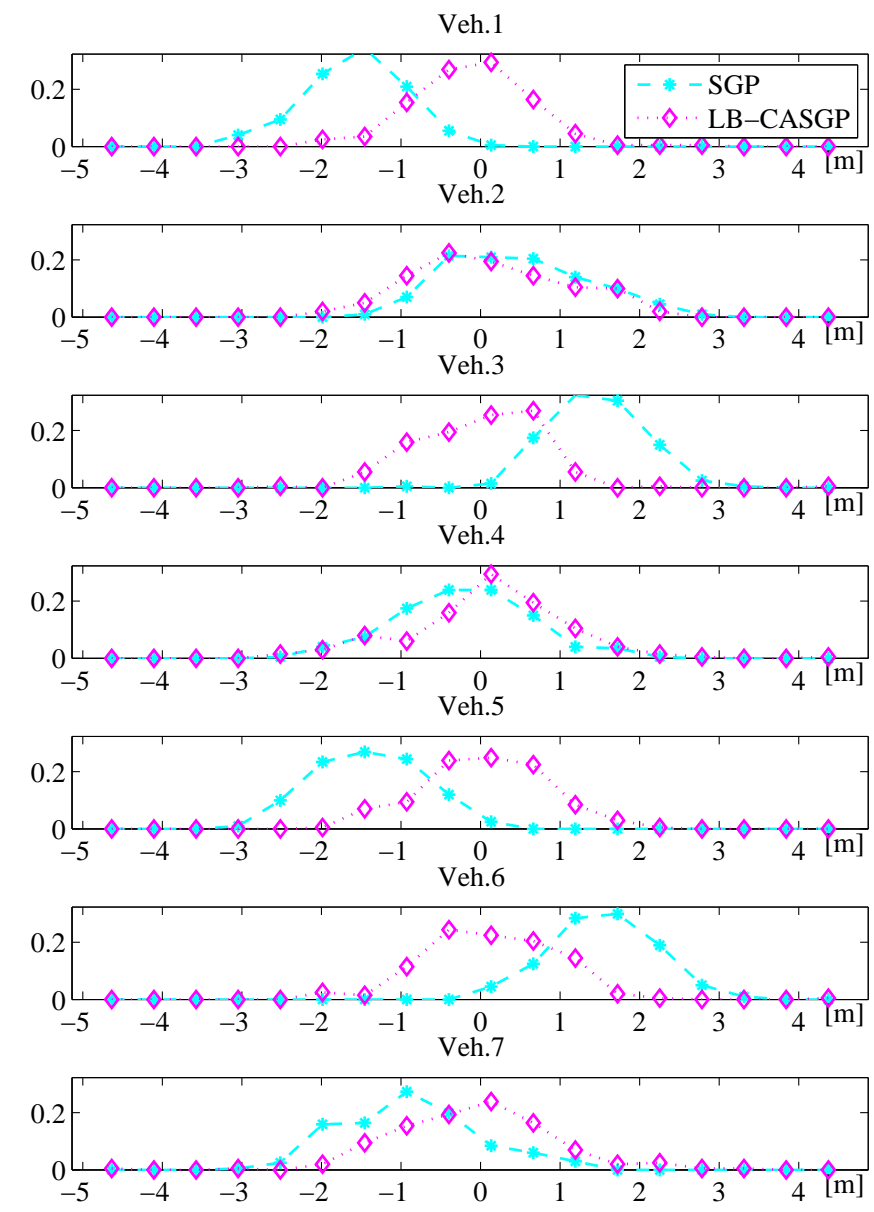

(b)

Figure 5: Simulation - normalized positioning error distribution, for all trajectory path for vehicles 1 to 7 , using $S G P$ and LB-CASGP estimation: (a) lateral, (b) longitudinal.

The small positive bias along the local coordinates $Y$-axis is not easy to observe on Figure 5 . The absolute mean lateral error value along the local coordinates $X$-axis of vehicles $1,3,5$ and 6 is therefore small, as well as the absolute mean longitudinal error value along the local coordinates $Y$-axis of vehicles 2 and 4 (see Table 3 ).

Regarding the real experiments results, from Figure 6 it is not possible to observe a predominant axis error, since the paths followed by all the vehicles have a closed loop shape. The absolute mean lateral and longitudinal error of $(S G P)$ for all the vehicles is quite moderate (see Table 3 ).

Figures 7 and Figure 8 display the normalized positioning lateral (top), longitudinal (center) and 2D euclidean (bottom) errors distributions with their associated cumulative distribution functions, for the simulated and real experiments respectively. The dashed line with '*' markers represents the position errors using $(S G P)$.

From the simulations results given in the top subplots of Figure 7 and in Table 3, one can observe that the algorithm has a very small mean lateral and longitudinal error distribution and a high standard deviation $\sigma$. This distribution profile is due to the fact that vehicles traveling in different directions have opposite error signals (see Figure 4(c)). The cumulative distribution $3 \sigma$ boundary of the lateral positioning error for $S G P$ algorithm is very high meaning that the positioning method is rather inaccurate most of the time.

From the center subplots of Figure 7 and in Table 3, one can observe that the standalone algorithm has nearly a zero mean longitudinal bimodal error distribution and a high standard deviation $\sigma$. This distribution profile is due to the fact that vehicles traveling in different directions have opposite error signals (see Figure 4(c)). The cumulative distribution $3 \sigma$ boundary of the longitudinal positioning error for $S G P$ algorithm is very high.

The bottom subplots of Figures 7 present the 2D euclidean error distribution. The cumulative distribution 


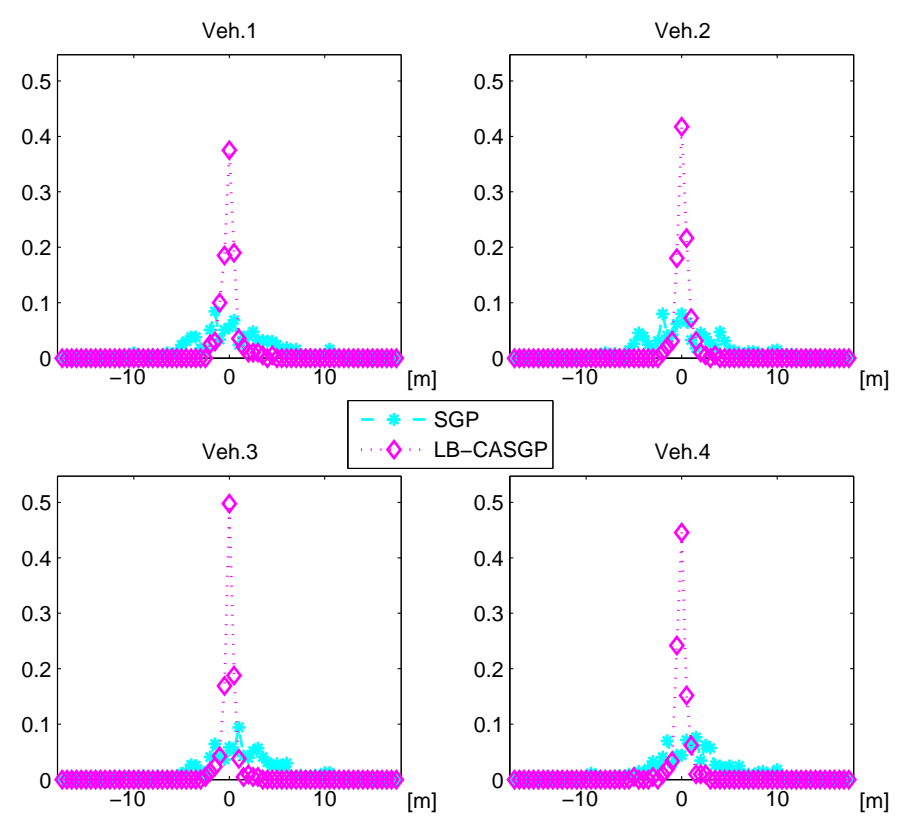

(a)
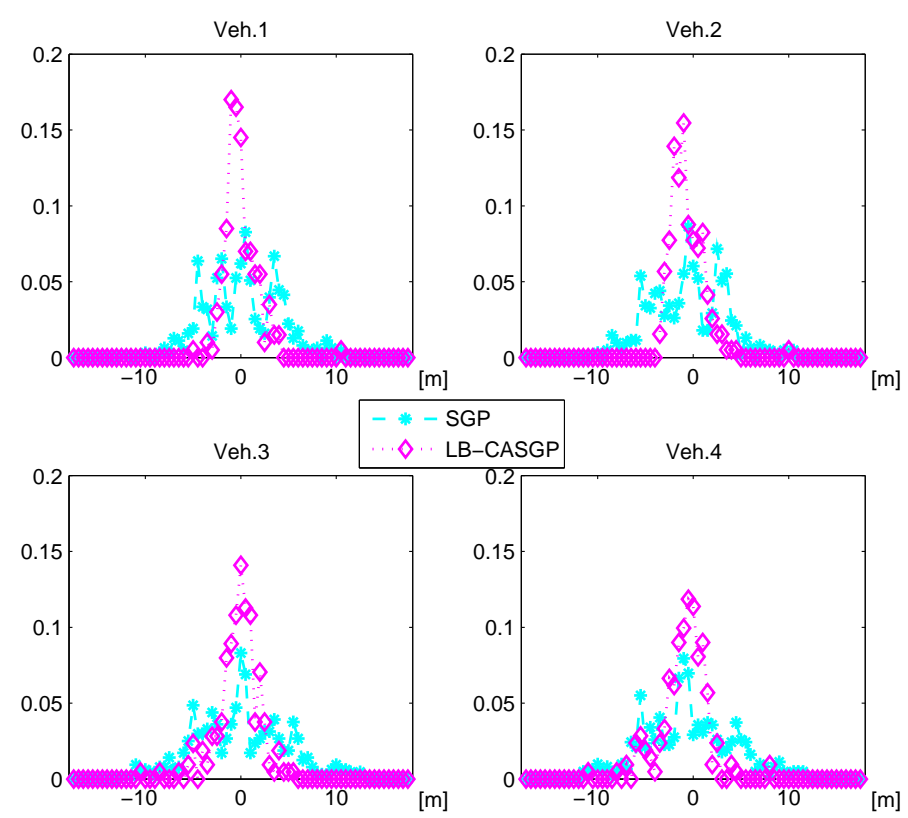

(b)

Figure 6: Real Experiments - normalized positioning error distribution, for all trajectory path for vehicles 1 to 4 , using $S G P$ and $L B-C A S G P$ estimation: (a) lateral, (b) longitudinal.

Table 3: ERror ANALYSIS FOR ALL VEHICLES.

\begin{tabular}{|c|c|c|c|c|c|c|c|c|c|c|c|c|c|c|}
\hline \multirow{2}{*}{\multicolumn{2}{|c|}{ Error [m] }} & \multicolumn{5}{|c|}{ Lateral } & \multicolumn{5}{|c|}{ Longitudinal } & \multicolumn{3}{|c|}{ Euclidean } \\
\hline & & ${ }^{b} \sum_{i=1}^{N}$ & $\frac{\left\|\overline{L A T_{i}}\right\|}{N}$ & mean & $\sigma$ & $3 \sigma$ & ${ }^{b} \sum_{i=1}^{N}$ & $\left\|\overline{L O N_{i}}\right\|$ & mean & $\sigma$ & $3 \sigma$ & mean & $\sigma$ & $3 \sigma$ \\
\hline \multirow{2}{*}{ Sim. } & vehicles & {$[1,3,5,6]$} & {$[2,4]$} & [1-7] & {$[1-7]$} & [1-7] & {$[1,3,5,6]$} & {$[2,4]$} & [1-7] & {$[1-7]$} & {$[1-7]$} & [1-7] & [1-7] & {$[1-7]$} \\
\hline & SGP & 0.372 & 1.558 & 0.204 & 1.27 & 3.300 & 1.494 & 0.349 & -0.092 & 1.402 & 3.187 & 1.803 & 0.624 & 3.633 \\
\hline \multirow{3}{*}{ Real } & vehicles & {$[1-$} & & [1-4] & [1-4] & [1-4] & [1-4] & [1-4] & [1-4] & {$[1-4]$} & {$[1-4]$} & [1-4] & [1-4] & {$[1-4]$} \\
\hline & SGP & 0.7 & & 0.701 & 4.367 & 13.45 & \multicolumn{2}{|c|}{0.301} & -0.234 & 4.301 & 12.38 & 5.324 & 3.125 & 13.95 \\
\hline & LB-CASGP & 0.0 & & 0.003 & 0.734 & 3.617 & \multicolumn{2}{|c|}{0.453} & -0.332 & 2.109 & 10.30 & 1.760 & 1.447 & 10.33 \\
\hline
\end{tabular}

${ }^{b} \sum_{i=1}^{N} \frac{\left\|\overline{L A T_{i}}\right\|}{N}$ and $\sum_{i=1}^{N} \frac{\left\|\overline{L O N_{i}}\right\|}{N}$ are the average of the absolute mean lateral and longitudinal error respectively.

$N$ represent the number of vehicles. $\overline{L A T_{i}}$ and $\overline{L O N_{i}}$ represent the mean lateral and longitudinal error of the ith vehicle respectively

$3 \sigma$ boundary of the $2 \mathrm{D}$ euclidean error is very high (see Table 3 ).

The real results depicted in Figure 8 and in Table 3 confirm the observations made in simulation for the $S G P$ algorithm: The lateral and longitudinal errors are quite large and highly spread.

\subsection{Cooperative Set-membership augmented GNSS positioning (LB-CASGP)}

The method presented in this section makes use of the lane-boundary measurements and exploits crosstrack corrections shared among networked vehicles in order to improve the performance by using the cooperative set-membership GNSS positioning method $L B-C A S G P$.

After having applied the lane-boundary constraint on the estimated set $\mathbb{X}$, a punctual position estimate $\left[x_{r}, y_{r}\right]$ is determined by projecting the solution of the center of gravity as explained before.

Figures 5 and 6 present the distributions of the lateral and longitudinal errors. In figures 7 and 8 , the dotted line with ' $\diamond$ ' marker, represent the position error of the $L B-C A S G P$ algorithm.

Figures 5 and 6 show clearly that the $L B-C A S G P$ concentrates the majority of both lateral and longitudinal errors around 0, while in $S G P$ the errors are more spread. This reveals that by sharing corrections, position estimates are improved in both the cross-track and the along-track direction.

From top subplots of Figures 7 and 8 and in Table 3, one can observe that the algorithm has nearly zero mean lateral error distribution and a very low standard deviation $\sigma$ which is due to the inclusion of the lane-boundary constraints provided by the GIS and the lane sensor. 

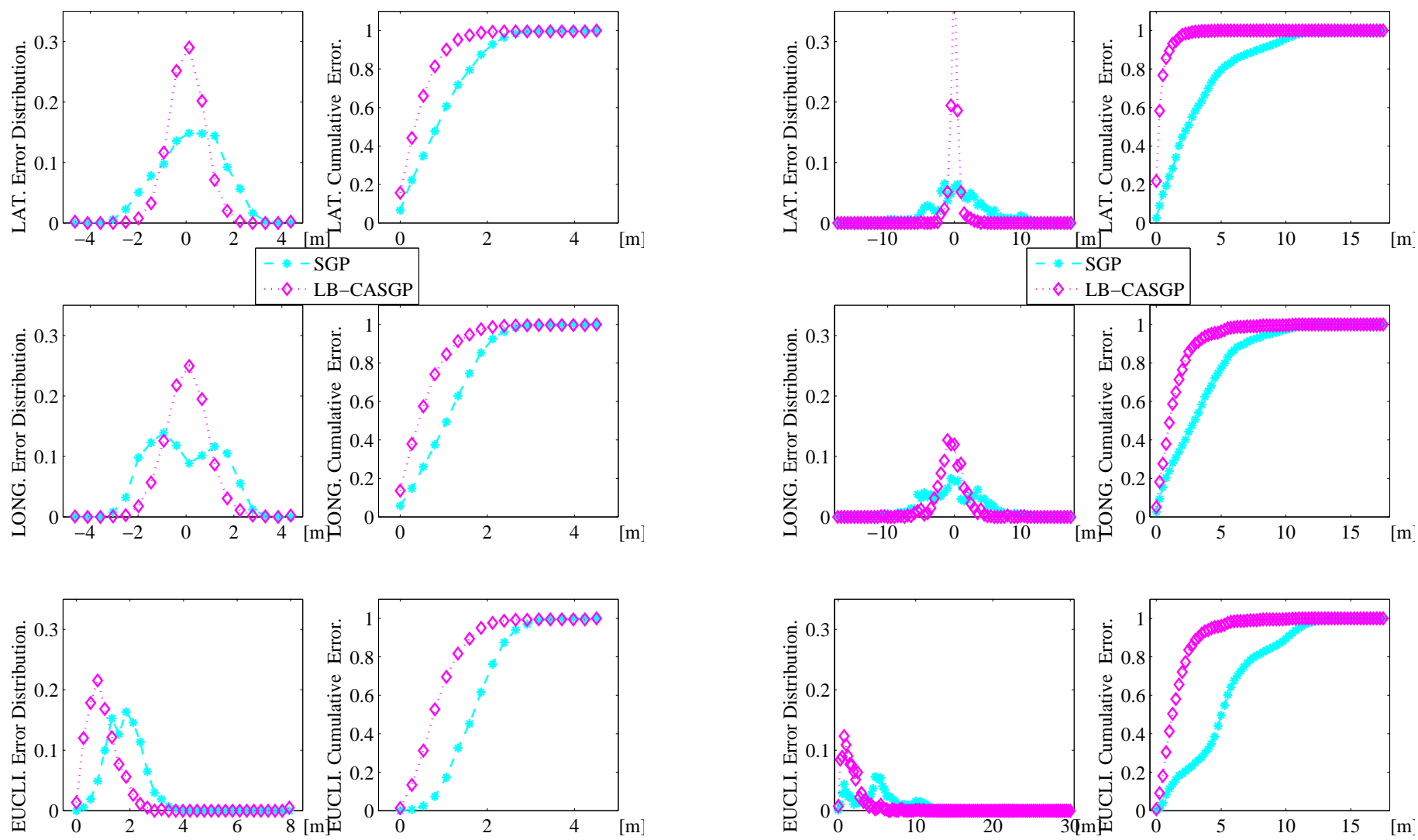

Figure 7: Simulation - All vehicles positioning errors, lateral (top), longitudinal (center) and 2D euclidean (bottom), using $S G P$ and $L B-C A S G P$ estimation: (a) normalized positioning errors distribution. (b) cumulative distribution functions.

Figure 8: Real Experiments - All vehicles positioning errors, lateral (top), longitudinal (center) and 2D euclidean (bottom), using $S G P$ and $L B-C A S G P$ estimation: (a) normalized positioning errors distribution. (b) cumulative distribution functions.

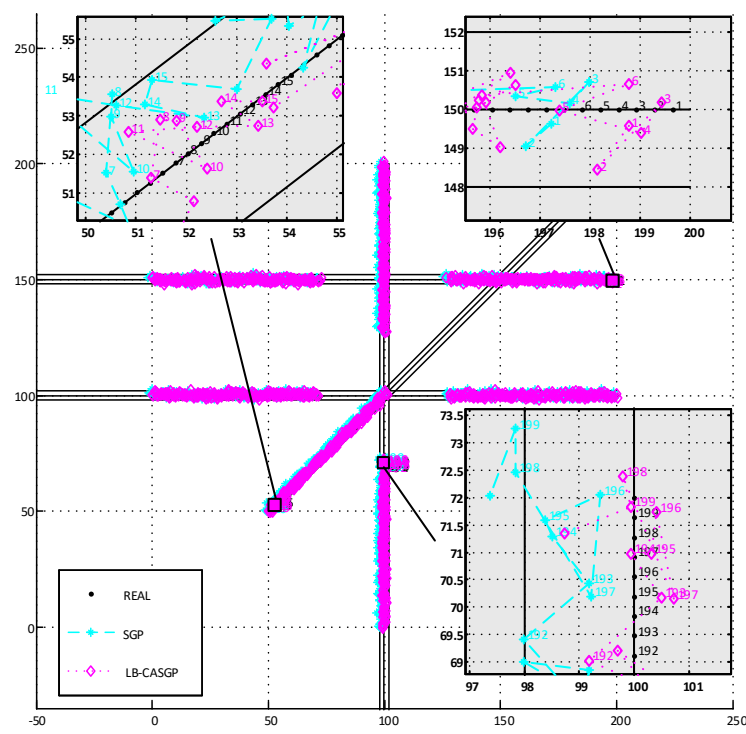

(a)

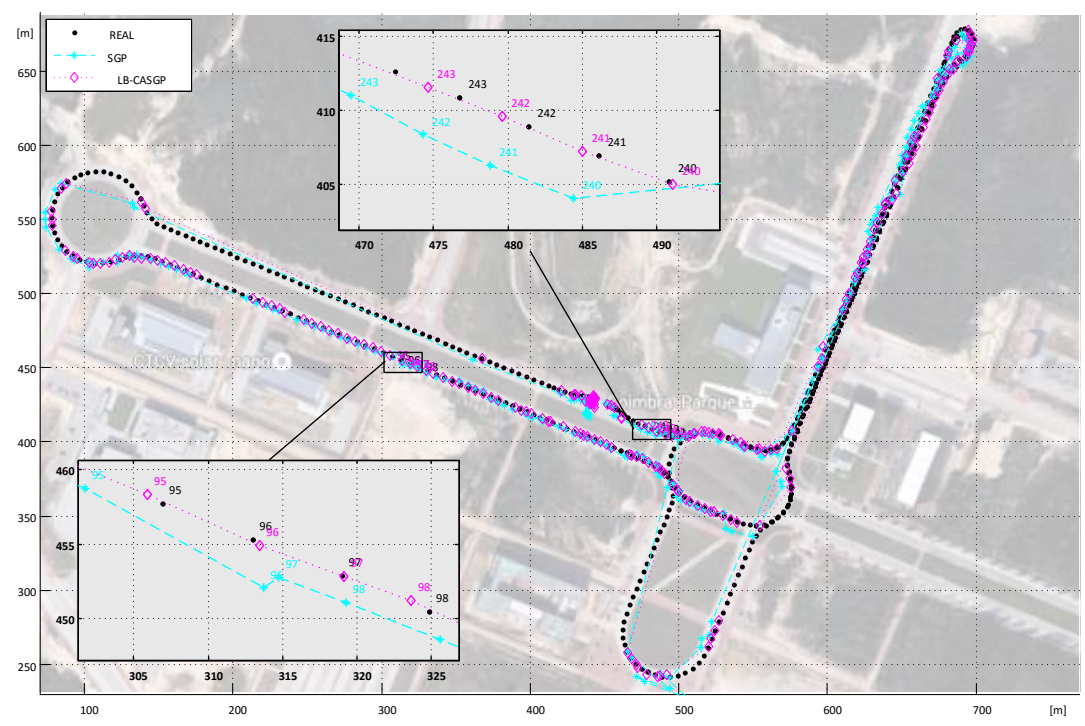

(b)

Figure 9: Trajectory and zoomed trajectory followed by each vehicle: (a) Simulation - vehicle 2 trajectory in the bottom-right zoom subplot; vehicle 6 trajectory in the top-right zoom subplot and vehicle 7 trajectory in the top-left zoom subplot (b) Real Experiments 

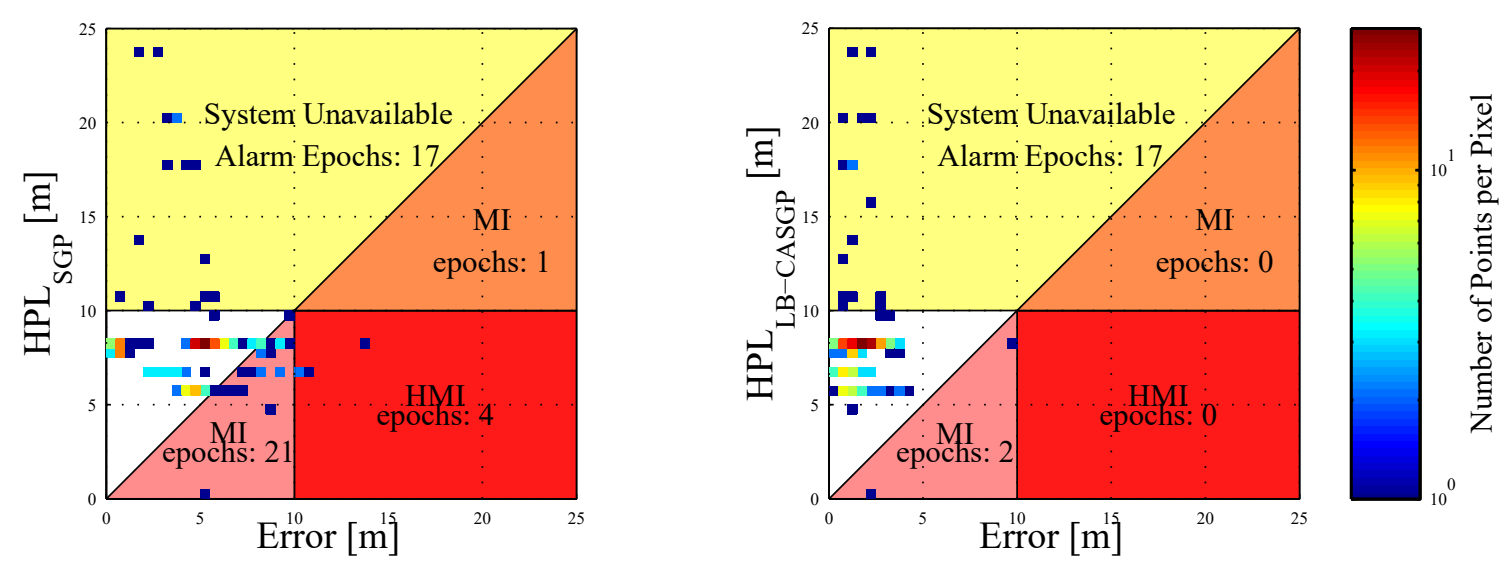

Figure 10: Real Experiments - HPL Stanford diagram: misleading information (MI), hazardously misleading information (HMI)

In terms of mean absolute lateral error, the $L B-C A S G P$ method provides an error reduction of at least $90 \%$ compared to $S G P$. The cumulative distribution $\sigma$ boundary of the lateral positioning error for $L B-C A S G P$ algorithm is greatly reduced meaning that the positioning method has an accuracy better than 1 meter both in simulation and in the real experiments. The $3 \sigma$ is reduced by least $60 \%$ when using the $L B-C A S G P$ algorithm, which mean a significant lateral error reduction during most of the time.

From center the subplots of Figures 7 and 8 and from Table 3, one can observe that algorithm has nearly zero mean longitudinal spread error distribution and a low standard deviation $\sigma$. As expected, when using $L B$ CASGP algorithm, the $\sigma$ is improved w.r.t. to the one obtained without the cooperative sharing of satellite specific errors, since the lane-boundary sensors not only provide additional geometric diversity for the axis orthogonal to the road lane of the carrying vehicle but as well for networked vehicles. The $\sigma$ is reduced by at least $49 \%$ when using the $L B-C A S G P$ algorithm, which is a significant longitudinal error reduction. The cumulative distribution $3 \sigma$ boundary of the longitudinal positioning error is also reduced as expected. The bottom subplots of Figures 7 and 8 display the 2D euclidean error distribution. For both simulations and real experiments, the mean and standard deviation $\sigma$ error of the $L B-C A S G P$ algorithm are reduced, being more evident on the real experiment and therefore validating the simulations.

The subplots of Figure 9(a), represent the zoomed trajectory for vehicles 2, 6 and 7, respectively bottomright, top-right and top-left zoom subplot. As mentioned earlier, this satellite constellation configuration generates errors along both $X$ and $Y$ local coordinates axes, with the major error axis along the negative direction of the local coordinates $X$-axis (see position estimation of vehicles 2 using $S G P$ in bottom-right zoomed zoomed of Figure 9(a)). Therefore, the cross-track improvement of position estimation by using $L B$ $C A S G P$ algorithm is more noticeable for vehicles travelling along the local coordinates $Y$-axis and with the lane-boundary sensors measurements along the local coordinates $X$-axis, as with vehicles 2 and 4 . The crosstrack position estimation improvement of $L B-C A S G P$ algorithm for vehicle 2 is shown in subplot bottom-right, where the position estimate is shifted right towards the real position. The along-track improvement of position estimation by using $L B-C A S G P$ algorithm is more noticeable for vehicles travelling along the local coordinates $X$-axis and using the shared corrections along the local coordinates $X$-axis, as with vehicles $1,3,5$ and 6 . The cross-track position estimation improvement of $L B-C A S G P$ algorithm for vehicle 6 is shown in subplot top-right, where the position estimate is shifted right towards the real position. The cross-track improvement for vehicles 1, 3, 5 and 6 and the along-track improvement for vehicles 2 and 4 is less evident since this satellite constellation produces a very small positive bias along the local coordinates $Y$-axis.

The position estimation for vehicle 7 reflects a medium improvement of $L B$-CASGP algorithm on the position estimate as this vehicle is not equipped with lane-boundary sensor. Nevertheless it benefits from the corrections estimates broadcast by all lane-boundary sensor equipped vehicles, making its correction noticeable in both cross-track and along-track. This correction can be observed on subplot top-left of Figure 9 (a).

The real experiment results shown in Figure 9(b), represent the trajectory followed by one of the four GPS receivers and its position estimation for each presented algorithm. Vehicles were platooning and therefore most of the time they were nearly collinear which is a situation closed to the singularity mentioned before. 
Remember that when all the networked vehicles are collinear, estimation is achieved using the noncooperative position method $L B-A S G P$. In Figure 9(b), only solutions when the $L B$-CASGP is able provide a solution are shown, i.e. only epochs where the vehicles are non-collinear or not nearly collinear are presented in the figure. By analysing the subplots of Figure 9(b), it is possible to observe that the satellite constellation configuration, during the real experiments, generates an error along the south-west direction (i.e. towards the top-right corner of Figure 9(b)) and west (i.e. towards the right side of Figure 9(b)). These biases are successfully mitigated using the $L B-C A S G P$ algorithm. The cross-track error is significantly removed as the position estimate is shifted towards north-east. The along-track error is corrected by shifting the position towards east. Subplots of Figure 9(b), where each position estimate is numbered, enables us to conclude that the position estimate using $L B-C A S G P$ is always better than using $S G P$, both in cross-track and in along-track. Although vehicle 7 is not equipped with a lane-boundary sensor, its position accuracy is improved in both cross-track and along-track directions, using the estimate of the satellite-specific errors $\left[C_{N, s s c} ; C_{E, s s c}\right]$, as described in equation 21. This improvement can be easily seen in the last row of Figure 5 a) and b).

\subsection{Integrity Analysis}

An integrity metric of major interest when dealing with integrity concerns is the Horizontal Protection Level (HPL) (Santa, 2006)(Tossaint , 2007). Stanford diagrams are widely used and consist of a 2D histogram of positioning solutions in terms of actual error and the estimated protection level. The Stanford diagrams of both $S G P$ and $L B-C A S G P$ obtained with the real data are shown in Figure 10. They illustrate the integrity performance achieved during the periods analyzed. In HPL the magnitude of the observed parity vector is used as a test statistic with the chi-squared distribution to detect single satellite failures. The HPL computation follows the following steps: determine a fault detection normalized threshold used in the Receiver Autonomous Integrity Monitoring (RAIM) computation, for a specified false alarm and degree of freedom; compute the probability density function of the chi-square and the the non-central chi-square distribution for specified degrees of freedom; determine the the parity vector associated with the over-determined user position solution and use it in the linear magnification (also referred in the literature as slope) between the horizontal radial error and the pseudo-range residual test statistic to obtain the HPL. For the HPL, it was considered a probability of missed detection of 0.001 and a false alarm tolerance of $3.33333 \times 10^{-7}$, the horizontal alarm limit (HAL) was set here to $10[\mathrm{~m}]$ but this value can be easily adapted to the requirements of different applications. Regarding the horizontal GNSS positioning system, its integrity risk is the probability that, at any moment, the horizontal position error (HPE) exceeds an HAL. The integrity system is declared unavailable when the HPL is greater than HAL. If the system is available and the HPE is not bounded by the HPL, the event is considered as a MI, since the HPL is always supposed to be an upper bound of the HPE. Moreover, the event is declared as HMI if the HPE exceeds the HAL (Tossaint, 2007).

The position error is not always bounded by the protection level, that is, there are several misleading information (MI) events in both algorithms, although they are almost ten times higher for the $S G P$. Regarding hazardously misleading information, the $L B-C A S G P$ achieves none events while the $S G P$ has four events. The real experiments results reveal that the $S G P$ algorithm is $78 \%$ of the time under normal operation whereas the $L B-C A S G P$ is $90 \%$, which is a significant improvement in terms of positioning availability.

\section{Conclusion}

This paper has presented a new cooperative positioning algorithm (LB-CASGP) based on constraints propagation on real intervals. This method improves the performance of standalone GNSS algorithms in environments where a lane-boundary sensor is capable of providing lateral corrections by detecting the distance from the vehicle to the lane. By sharing their corrections, networked vehicles can improve their own estimates and assist the others.

The errors magnitude and the confidence domains have been evaluated in a comparative way both in simulation and through real experiments with the same software implementation. As the implementation of both set-inversion methods based algorithms is quite straightforward, the resulting code is simple and compact.

When appliyng the $L B-C A S G P$ algorithm, the satellite-specific errors are used in a cooperative framework enabling its reduction on networked vehicles in both cross-track and along-track components. This method 
can also improve position estimates of vehicles not equipped with lane-boundary sensor. The non-equipped vehicles use the cooperative estimation of the satellite-specific errors to improve its own position estimate.

Both set-inversion algorithms (i.e. $S G P$ and $L B-C A S G P$ ) have the advantage of guaranteeing not to lose any solution in the computation process and are insensitive to local minimum convergence issues. They are therefore naturally very reliable by nature. The risk of the positioning solution set not to include the real position is solely related to the risk taken when selecting the bounds parameters on the measurements. When subjected to multipath disturbances the $L B-C A S G P$ can provide worst estimation than stand-alone solutions, i.e. the multipath subjected vehicle is contaminating the estimation of the vehicles in the vicinity. Therefore, further work is being carried on extending the guaranteed zone computation algorithm to take into account outliers, namely, using a relaxed set-membership approach.

\section{Acknowledgment}

This work has been supported by Fundação para a Ciência e Tecnologia (FCT) through project grant AMSHMI12: RECI/EEIAUT/ 0181/2012, cofounded by FEDER (COMPETE program). We also acknowledge the support of QREN Project B-Mobility4People (maisCENTRO program).

\section{References}

Alonso11 Alonso, J., Milanés, V., Pérez, J., Onieva, E., González, C., De Pedro, T., Autonomous vehicle control systems for safe crossroads, Transportation Research part C: emerging technologies, 19(6), 1095-1110, 2011.

Santa, J., Toledo-Moreo, R., Zamora-Izquierdo, M. A., Úbeda, B., Gómez-Skarmeta, A. F., An analysis of communication and navigation issues in collision avoidance support systems, Transportation Research part C: emerging technologies, 18(3), 351-366, 2010.

Choi, S. K., An ontological model to support communications of situation-aware vehicles, Transportation Research Part C: Emerging Technologies, 53, 112-133, 2015.

Drevelle, V., Bonnifait, P., Integrity zone computation using interval analysis, European Navigation ConferenceGlobal Navigation Satellite Systems (ENC-GNSS), 2009.

Toledo-Moreo, R., Zamora-Izquierdo, M. A., Collision avoidance support in roads with lateral and longitudinal maneuver prediction by fusing GPS/IMU and digital maps, Transportation Research part C: emerging technologies, 18(4), 611-625, 2010.

Sun, R., Ochieng, W. Y., Feng, S., An integrated solution for lane level irregular driving detection on highways, Transportation Research Part C: Emerging Technologies, 56, 61-79, 2015.

N.M. Drawil and O. Basir, Intervehicle-communication-assisted localization, IEEE Transactions on Intelligent Transportation Systems, vol.11, no.3, pp.678-691, Sept. 2010.

Williams, T., Alves, P., Lachapelle, G., Basnayake, C., Evaluation of GPS-based methods of relative positioning for automotive safety applications. Transportation research part C: emerging technologies, 23, 98-108, 2012.

M. Woo, J. Choi, H. Han, Carrier phase GPS/millimeter-wave radar for vehicle platooning, IEEE Int. Symp. on Industrial Electronics (ISIE), vol.3, pp.1548-1552 vol.3, 2001.

G. Challita, S. Mousset, F. Nashashibi and A. Bensrhair, An application of V2V communications: cooperation of vehicles for a better car tracking using GPS and vision systems, IEEE Vehicular Networking Conference (VNC), pp.1-6, 28-30 Oct. 2009.

J. Hur, S. N. Kang and S. W. Seo, Multi-lane detection in urban driving environments using conditional random fields, IEEE Intelligent Vehicles Symposium (IV), 2013. 
Rife, J., Xiao, X., Estimation of Spatially Correlated Errors in Vehicular Collaborative Navigation with Shared GNSS and Road-Boundary Measurements, Int. Tech. Meeting of The Satellite Division of the Institute of Navigation (ION GNSS 2010), Portland, pp. 1667-1677, Sept. 2010.

J. Rife, Collaborative vision-integrated pseudorange error removal: team-estimated differential GNSS corrections with no stationary reference receiver, IEEE Transactions on Intelligent Transportation Systems, vol.13, no.1, pp.15-24, March 2012.

Drevelle, V., Bonnifait, P., Localization Confidence Domains via Set Inversion on Short-Term Trajectory, IEEE Transactions on Robotics, vol.29, no.5, pp.1244,1256, Oct. 2013

Elliott D. Kaplan, Understanding GPS: Principles and Applications, Artech House Publishers, Boston, 1996.

L. Jaulin, M. Kieffer, O. Didrit, and E. Walter, Applied Interval Analysis, New York: Springer-Verlag, 2001.

Tornil-Sin, S., V. Puig, and T. Escobet.; Set computations with subpavings in MATLAB: The SCS Toolbox, IEEE Int. Symp. on Computer-Aided Control System Design (CACSD), 2010.

L. Jaulin and E. Walter, Guaranteed nonlinear estimation and robust stability analysis viaset inversion, Proc. of the 2rd European Control Conference (ECC), Groningen, Netherlands, 1993.

L.C.Bento, R.Parafita, S.Santos and U.Nunes, An Intelligent Traffic Management at Intersections legacy mode for vehicles not equipped with V2V and V2I Communications, IEEE Int.Conf. Intelligent Transportation Systems, Hague, Netherlands, 2013.

Urbano Nunes and L. Conde Bento, Data fusion and path-following controllers comparison for autonomous vehicles, in Nonlinear dynamics Journal, Springer-Verlag, Vol.49, pp.445-462, Sept. 2007.

Tetewsky, Avram K., and Arnold Soltz, GPS Matlab Toolbox Review, GPS World 9, no. 10 (1998): 50-57.

Gat, Itay, Meny Benady, and Amnon Shashua, A monocular vision advance warning system for the automotive aftermarket, No.2005-01-1470, SAE Technical Paper, 2005.

Santa, J.; Ubeda, B.; Toledo, R.; Skarmeta, A.F.G., Monitoring the Position Integrity in Road Transport Localization Based Services, IEEE 64th Vehicular Technology Conference (VTC-2006), Sept. 2006.

Tossaint, M., et al. The Stanford-ESA Integrity Diagram, Journal of the Institute of Navigation (ION), Vol.54, No.2, 2007. 